\title{
Doppler shifts and spectral line profile changes in the starlight scattered from an exoplanet
}

\author{
John B. P. Strachan ${ }^{\circledR \star}$ and Guillem Anglada-Escudé \\ School of Physics and Astronomy, Queen Mary University of London, 327 Mile End Rd., London E1 4NS, UK
}

Accepted 2020 January 17. Received 2020 January 15; in original form 2019 July 11

\begin{abstract}
Scattered starlight from an exoplanet, commonly called reflected light, can be used to characterize the planet including the determination of its albedo and inclination of orbit. The relatively low flux ratio between directly observed starlight and starlight scattered off hot Jupiters make these systems the prime candidates for detection of reflected light using high-resolution spectroscopy. The first detections have been claimed for $51 \mathrm{Peg} \mathrm{b}$. In a first calculation, we derive the Doppler shift of reflected light measured by a remote observer for a planet modelled as a point-like particle in orbit around a star. We find that the Doppler shift of reflected light from planets have a different Doppler shift to that of light emitted directly from the planet with magnitude equivalent to the radial motion of the planet with respect to the star. This only occurs for non-circular orbits. Secondly, restricting our investigation to planets that are tidally locked and orbit in circular orbits we account for the finite size of the star and planet by integrating the contribution to a simulated spectral line across both their surfaces. Since exact analytical expressions cannot be easily derived as a function of all free parameters of the problem, we have developed a software tool called REFLECTED STARLIGHT (RESTART) that produces the resulting line profiles. By applying it to study cases found in the literature, we explicitly show that hot Jupiters such as WASP-19b and 51 Peg b should show substantial broadening and asymmetric distortions compared to the nominal stellar line.
\end{abstract}

Key words: techniques: spectroscopic-planet-star interactions-stars: rotation.

\section{INTRODUCTION}

Since the detection of the first exoplanet orbiting a main-sequence star in 1995 (Mayor \& Queloz 1995), there are now over 4100 planet candidates with more than 400 of these being either hot or very hot Jupiters according to the Extrasolar Planet Encyclopaedia (Schneider et al. 2011). Hot Jupiters are defined as planets with mass approximately that of Jupiter (here, we assume greater than 100 Earth masses) and that orbit in a period of 3-9d and have a semimajor axis less than $0.1 \mathrm{au}$. Very hot Jupiters orbit in period $\mathrm{P}$ $<3$ d. (Perryman 2018).

Reflected light is the light scattered off the planet originating from its parent star. Reflected light is readily observed in the planets of the Solar system. The continuum of a typical spectrum of a Solar system planet has two peaks relating to where the reflected light dominates the spectrum at visible wavelengths and where thermal emission dominates in the near-infrared. Models derived for the spectrum of hot Jupiters also show the same peaks (Seager 2010).

^E-mail: j.b.p.strachan@qmul.ac.uk
Detecting reflected light is difficult. Even for planets in the solar neighbourhood there is a very small angle separating the planet from its parent star. This makes it difficult to separate the light from the planet and its parent star. This difficulty is compounded by the extremely small flux ratio between them (Claudi 2016). The largest flux ratios are expected for hot Jupiters and to be of the order of a few parts in $10^{-5}$ and is due to the large size of the planet and its proximity to its parent star. For an Earth twin orbiting a Sun-like star, a contrast ratio is expected of the order $10^{-10}$ (Martins et al. 2016).

High-resolution spectroscopy is seen as a potentially advantageous method of detecting reflected light, as although light scattered from the planet is expected to have a similar spectrum to the starlight (Seager \& Sasselov 1998) it can be disentangled from the spectrum of the star in velocity space due to the large Doppler shift of the planet with respect to the star. Various techniques have been used to identify the signal of the reflected light including most recently using cross-correlation functions (CCFs) by Martins et al. (2015) or autocorrelation functions (ACFs) by Borra \& Deschatelets (2018) on each spectrum. These techniques effectively combine the lines in the planet spectrum in order to increase the signal to noise. The CCFs (or ACFs) are then moved to the frame of the planet and combined 
in order to increase the signal to noise further to a level where the signal from the reflected light can be detected. If no detection can be made, then an upper bound on the strength of the reflected light signal can be given. In order to know the wavelength position of the reflected light in the spectrum, the required orbital parameters of the orbit need to be known in advance and come from the transit and radial velocity $(\mathrm{RV})$ planet detection methods. In the case where the planet does not transit then the inclination parameter has to be fitted whilst performing the search for the reflected light. Indeed, detecting the reflected light for a system without a previously known inclination is important as once identified the mass of the planet can then be determined (Charbonneau et al. 1999).

Photometry can also be used to detect reflected light. Light curves with high enough signal to noise should be able to show the increase in light towards the superior conjunction and decrease towards inferior conjunction. The light curves of $v$ And $b$ in the mid-infrared (Harrington et al. 2006) and HAT-P-7b in the visible (Borucki et al. 2009) provide examples of this. From the detection of reflected light in photometry, the flux ratio can be determined and provide input to the required signal to noise required to detect the reflected light in high-resolution spectroscopy.

The 51 Peg system is the only system where high-resolution spectroscopic detection of reflected light has been claimed in the optical, where Martins et al. (2015) used the CCF method and Borra \& Deschatelets (2018) used the ACF method. Contrast levels were of the order of $6 \times 10^{-5}$ and significant broadening was observed in the CCF line profile. High-precision photometry from MOST (Walker et al. 2006) of 51 Peg shows a light curve that peaks just before orbital phase 0.1 at a level of $2.5 \times 10^{-4}$ greater than the minimum in the light curve.

In this paper, we determine the Doppler shift of starlight scattered off of exoplanets and show how for hot Jupiters the scattering can result in significant broadening of the spectral line profiles. We have produced a tool called Reflected STARlighT (RESTART) that will produce a template spectrum of the reflected light from the planet based on the spectra from the star.

In Section 2 of this paper, we describe analytical models for reflected light and also describe the code RESTART that models reflected light spectra.

In Section 3, we provide results from running RESTART for the examples of the very hot Jupiter WASP-19b, the hot Jupiter 51 Peg $\mathrm{b}$, and an Earth analogue system where the system is $10 \mathrm{pc}$ from the observer. We compare our results with what we expect and from the detection paper for $51 \mathrm{Peg}$ b.

In Section 4, we give the discussion and in Section 5 the conclusions to the paper. Appendices A-C provide derivations of some of the results that are referred to in Section 2.

\section{ANALYTICAL MODEL AND THE RESTART CODE}

We first determine the Doppler shift formula in a suitable form for starlight reflected off of a particle (Section 2.1). Then, using this equation we compute the Doppler shift of reflected light in a two-body star/planet system where the planet and the star are far enough away from each other that they can both be considered to be point-like (Section 2.2). We then model the flux of reflected starlight from a star and planet two-body system where the size of the planet and the star has to be taken into account due to their proximity. We restrict the orbits in this system to be circular and the planet to be tidally locked that are expected for hot Jupiter systems (Section 2.3).
The final expressions for the flux from Section 2.3 cannot be easily solved analytically as a function of all the parameters of the problem. Thus, we describe our software implementation called RESTART of these equations in Section 2.4, where we take as input the rotationally unbroadened spectrum from a star and have as output the spectrum of the reflected starlight as seen by a distant observer.

\subsection{Doppler shift of light reflected from a point-like particle}

In order to detect scattered starlight from an exoplanet, we need to be able to determine the Doppler shift of the reflected light as viewed by the observer on the Earth. The non-relativistic Doppler equation commonly used for reflection off of a mirror from Anglada-Escudé et al. (2007) is

$v^{\prime}=v\left[1-2 \frac{1}{c}\left((\hat{\boldsymbol{\sigma}} \cdot \hat{\boldsymbol{n}})\left(\boldsymbol{v}_{m} \cdot \hat{\boldsymbol{n}}\right)\right)\right]$,

where $\mathrm{c}$ is the speed of light, $v^{\prime}$ is the Doppler shifted frequency, $v$ is the original frequency, $\hat{\sigma}$ is the unit vector of the incoming light ray, $\hat{\mathbf{n}}$ is the normal to the mirror, and $\mathbf{v}_{\mathbf{m}}$ is the velocity the mirror.

In the case of light scattering off of a particle, we take the mirror to be perpendicular to the bisector between the incoming light ray and the outgoing scattered ray. Thus, $\hat{\mathbf{n}}$ is

$\hat{\boldsymbol{n}}=\frac{\hat{\sigma}^{\prime}-\hat{\sigma}}{\left|\hat{\sigma}^{\prime}-\hat{\sigma}\right|}$

where $\hat{\sigma}^{\prime}$ is the unit vector of the outgoing ray.

Substituting this value for $\hat{\boldsymbol{n}}$ in equation (1) and denoting $\boldsymbol{v}_{\boldsymbol{p}}$ as the velocity of the particle, we have

$v^{\prime}=v+\frac{v}{c} v_{p} \cdot\left(\hat{\boldsymbol{\sigma}}^{\prime}-\hat{\boldsymbol{\sigma}}\right)$.

Setting $\Delta v=v^{\prime}-v$ and using the non-relativistic version of the Doppler shift equation

$\frac{\Delta v}{v}=\frac{\Delta \mathrm{v}}{c}$,

where $\Delta v$ is the Doppler shift of the outgoing ray in velocity space and then substituting for $\frac{\Delta v}{v}$ in equation (3) we have

$\Delta \mathrm{v}=v_{p} \cdot\left(\hat{\sigma}^{\prime}-\hat{\sigma}\right)$.

The Doppler shift of scattered light caused by moving particles as a non-invasive way of determining the velocity of fluids has been studied extensively (see Charrett, James \& Tatam 2012 for a review of laser Doppler velocimetry). Particles are inserted into the fluid (if the fluid itself is not reflective) and light rays usually using lasers are fired into the fluid. The light reflected is then captured on detectors and the frequency shift of the captured light is used to determine the velocity of the flows. The velocity flows studied range from small velocities to supersonic. In many cases, these particles are small and exhibit mainly Rayleigh scattering (Yeh \& Cummins 1964), which is the same type of scattering we may expect from hot Jupiters (Seager \& Sasselov 1998). It is very straightforward to show that the equations used to determine the velocity of the flows in the aforementioned papers are consistent with equation (3).

\subsection{Doppler Shift of reflected light from exoplanets modelled as point-like particles}

Here, we determine the Doppler shift of reflected light observed from the Earth of a star/planet system where the following assumptions hold: 


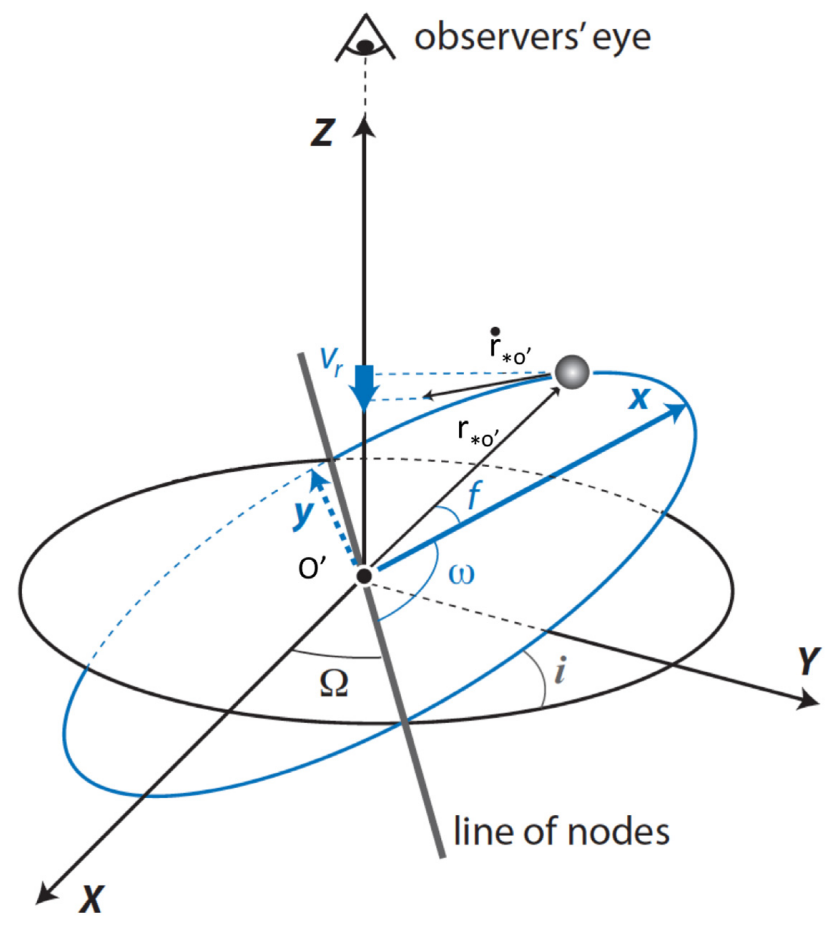

Figure 1. The relationship between the plane of the orbit of the star (blue) to the plane of the sky (black) adapted from Murray \& Correia (2010).

(i) The only gravitational influence on the planet in the system is the parent star, so we can assume a simple two-body problem.

(ii) The planet is far enough away from the star so that both objects can be treated as point masses and the starlight reaching the planet are in parallel rays

(iii) We ignore the effects of rotation of the planet and star.

We follow the two-body problem as described in Murray \& Correia (2010).

In the two-body problem, the position of the planet with respect to the star is typically first determined in a planar Cartesian coordinate system $x y$, which is in the plane of the orbit centred on the star. The coordinates of the planet are given by

$x=r \cos f$,

$y=r \sin f$,

where $\mathrm{r}$ is the distance from the planet to the star and $\mathrm{f}$ is the true anomaly (see Fig. 1).

These equations are then transformed to the Cartesian coordinate system for the orbit in space where the $X$ - and $Y$-axes are in the plane of the sky and the $Z$-axis points towards the observer. The position of the planet with respect to the star is then given by the following equations:

$X=r(\cos \Omega \cos (\omega+f)-\sin \Omega \sin (\omega+f) \cos I)$,

$Y=r(\sin \Omega \cos (\omega+f)+\cos \Omega \sin (\omega+f) \cos I)$

$Z=r \sin (\omega+f) \sin I$,

where the three angles involved in the transformation are the longitude of ascending node $\Omega$, argument of pericentre $\omega$, and inclination I. These angles are used to rotate the $x y$ axis, which

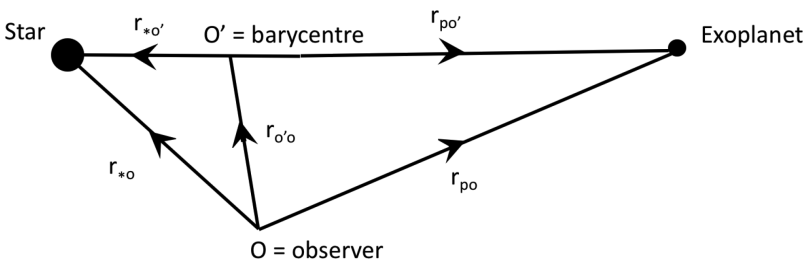

Figure 2. Position vectors to planet and star with respect to barycentre $\mathrm{O}^{\prime}$ and observer $\mathrm{O}$

is in the plane of the orbit, to the $X Y Z$ axis, which is the orbit in space.

The above three equations can be used to give the position of the planet with respect to the barycentre of the system if their right-hand sides are multiplied by the factor $\frac{m_{*}}{m_{*}+m_{p}}$, where $m_{\mathrm{p}}$ and $m_{*}$ are the mass of the planet and the star, respectively.

Similarly, the equations can be used to give the position of the star with respect to the centre of mass if their right-hand sides are multiplied by the factor $\frac{m_{p}}{m_{*}+m_{p}}$ and $\omega$ is replaced by $\omega_{*}$, where $\omega_{*}=$ $\omega+\pi$.

The equation for the velocity shift $\Delta \boldsymbol{v}$ due to the reflection is given in equation (5) where in this case $\hat{\sigma}$ is the

unit vector in the direction of the planet from the barycentre and $\hat{\sigma}^{\prime}$ is the unit vector from the planet to the observer. In the $X Y Z$ coordinate frame $\hat{\sigma}$ and $\hat{\sigma}^{\prime}$ are

$\hat{\boldsymbol{\sigma}}=\left[\begin{array}{c}\cos \Omega \cos (\omega+f)-\sin \Omega \sin (\omega+f) \cos I \\ \sin \Omega \cos (\omega+f)+\cos \Omega \sin (\omega+f) \cos I \\ \sin (\omega+f) \sin I\end{array}\right]$,

$\hat{\boldsymbol{\sigma}^{\prime}}=\left[\begin{array}{l}0 \\ 0 \\ 1\end{array}\right]$.

The position of the planet with respect to the observer is from Fig. 2:

$\boldsymbol{r}_{p o}=\boldsymbol{r}_{o^{\prime} o}+\boldsymbol{r}_{p o^{\prime}}$.

Differentiating, we get the velocity

$\dot{\boldsymbol{r}}_{p o}=\dot{\boldsymbol{r}}_{o^{\prime} o}+\dot{\boldsymbol{r}}_{p o^{\prime}}$.

The first term on the right is the barycentric contribution to the velocity with respect to the observer. The second term is the velocity of the planet with respect to the observer. We will work in the barycentric frame to determine the velocity shift due to the reflection in that frame and then move to the observer frame to get the shift due to the observer moving with respect to the barycentre.

The velocity of the planet with respect to the barycentre is

$\boldsymbol{v}_{\mathrm{p}, \mathrm{b}}=\dot{\boldsymbol{r}}_{p o^{\prime}}=\frac{m_{*}}{m_{*}+m_{\mathrm{p}}}\left[\begin{array}{c}\dot{X} \\ \dot{Y} \\ \dot{Z}\end{array}\right]$,

where

$$
\begin{aligned}
\dot{X}= & \dot{r}[\cos \Omega \cos (\omega+f)-\sin \Omega \sin (\omega+f) \cos I] \\
& -r \dot{f}[\cos \Omega \sin (\omega+f)+\sin \Omega \cos (\omega+f) \cos I], \\
\dot{Y}= & \dot{r}[\sin \Omega \cos (\omega+f)+\cos \Omega \sin (\omega+f) \cos I] \\
& +r \dot{f}[\cos \Omega \cos (\omega+f) \cos I-\sin \Omega \sin (\omega+f)],
\end{aligned}
$$


$\dot{Z}=\dot{r} \sin (\omega+f) \sin I+r \dot{f} \cos (\omega+f) \sin I$.

The Doppler shift in the barycentric frame of the reflected light is composed of two Doppler shifts. First, the Doppler shift when the light is emitted from the star and secondly from the reflection of the light off of the planet. The total Doppler shift is given by

$\Delta \boldsymbol{v}_{p, b}=-\frac{m_{\mathrm{p}}}{m_{*}+m_{\mathrm{p}}} \dot{\boldsymbol{r}}+\boldsymbol{v}_{\boldsymbol{p}, \boldsymbol{b}} \cdot\left(\hat{\boldsymbol{\sigma}}^{\prime}-\hat{\boldsymbol{\sigma}}\right)$.

From equations (11) and (12), we have

$\hat{\boldsymbol{\sigma}}^{\prime}-\hat{\boldsymbol{\sigma}}=\left[\begin{array}{c}-\cos \Omega \cos (\omega+f)+\sin \Omega \sin (\omega+f) \cos I \\ -\sin \Omega \cos (\omega+f)-\cos \Omega \sin (\omega+f) \cos I \\ 1-\sin (\omega+f) \sin I\end{array}\right]$,

and thus the total Doppler shift is

$$
\begin{aligned}
\Delta v_{p, b}= & -\frac{m_{\mathrm{p}}}{m_{*}+m_{\mathrm{p}}} \dot{r}+\left(\frac{m_{*}}{m_{*}+m_{\mathrm{p}}}\right) \\
& \times(\dot{r}[\sin (\omega+f) \sin I-1]+r \dot{f}[\cos (\omega+f) \sin I]) .
\end{aligned}
$$

Now, if we now consider the frame of the observer and assume the proper motion of the barycentre is $V_{z}$ then we have

$$
\begin{aligned}
\Delta v_{p, o}= & V_{z}-\frac{m_{\mathrm{p}}}{m_{*}+m_{\mathrm{p}}} \dot{r}+\left(\frac{m_{*}}{m_{*}+m_{\mathrm{p}}}\right) \\
& \times(\dot{r}[\sin (\omega+f) \sin I-1]+r \dot{f}[\cos (\omega+f) \sin I]) .
\end{aligned}
$$

From the point of view of the observer, the Doppler shift of light emitted from the planet is (using equation 14)

$\Delta v_{r, o}=\dot{\boldsymbol{r}}_{p o} \cdot \hat{z}=\dot{\boldsymbol{r}}_{o^{\prime} o} \cdot \hat{\boldsymbol{z}}+\dot{\boldsymbol{r}}_{p o^{\prime}} \cdot \hat{z}$,

where $\hat{z}=(0,0,1)^{T}$ and expanding out this equation using equations (15) and (18) we have

$$
\begin{aligned}
\Delta v_{r, o}= & V_{z}+\frac{m_{*}}{m_{*}+m_{\mathrm{p}}} \\
& \times(\dot{r} \sin (\omega+f) \sin I+r \dot{f} \cos (\omega+f) \sin I) .
\end{aligned}
$$

Thus, the difference between the Doppler shift of light reflected and light originating directly from the planet is given by

$\Delta v_{p, o}-\Delta v_{r, o}=-\dot{r}$,

where $\dot{r}$ is given in terms of eccentricity e, semimajor axis a, and mean motion $\mathrm{n}$ where

$n=\frac{2 \pi}{P}$,

and true anomaly $f$ by

$\dot{r}=\frac{n a}{\sqrt{1-e^{2}}} e \sin f$.

From the above equation, we can see that there will be a difference in observed Doppler shift between reflected starlight and light emitted directly from the planet when either the eccentricity is 0 - we have a circular orbit - or when the true anomaly is 0 .
An order-of-magnitude estimate of the size of $\dot{r}$ can be made by first observing that

$\dot{r} \leq \frac{n a e}{\sqrt{1-e^{2}}}$.

From Keplers third law, we have

$\mu=a^{3} n^{2}$,

where

$\mu=G\left(m_{\mathrm{p}}+m_{*}\right) \approx G m_{*}$

and thus

$\dot{r} \leq \frac{n^{1 / 3} \mu^{1 / 3} e}{\sqrt{1-e^{2}}}$.

The upper bound for $\dot{r}$ given above can be expressed in terms of the period and eccentricity of the planet, which we can obtain using the RV method and the mass of the star using equations (26), (29), and (30):

$\dot{r} \leq(2 \pi G)^{1 / 3} m_{*}^{1 / 3} P^{-1 / 3} \sqrt{\frac{e^{2}}{1-e^{2}}}$.

It is useful to express this equation in terms of more practical units:

$\dot{r} \leq 212.908 \mathrm{~km} \mathrm{~s}^{-1}\left(\frac{m_{*}}{M_{\odot}}\right)^{1 / 3}\left(\frac{P}{\text { day }}\right)^{-1 / 3} \sqrt{\frac{e^{2}}{1-e^{2}}}$.

When the eccentricity is small (e $<<1$ ), we can simplify the above equation to

$\dot{r} \leq 212.908 \mathrm{~km} \mathrm{~s}^{-1}\left(\frac{m_{*}}{M_{\odot}}\right)^{1 / 3}\left(\frac{P}{\text { day }}\right)^{-1 / 3} e$.

It is useful to observe that $\dot{r}$ is independent of the inclination of the orbit. An important implication of this is that high-resolution spectroscopy could now be used to detect exoplanets, via detection of the Doppler shift of reflected light, in low inclination, even face on orbits, provided the orbits were eccentric.

\subsection{Spectral line profile of reflected light from tidally locked hot Jupiters}

In this subsection, we outline how we determine the flux and thus spectral line profile of reflected light arriving at the observer on the Earth from a tidally locked hot Jupiter in a circular orbit. Full details of the calculations are given in Appendix A. In this case, our main assumptions are as follows:

(i) The only gravitational influence on the planet in the system is the parent star, so we can assume a simple two-body problem.

(ii) The tidal interactions between planet and star have caused the orbits around the barycentre to be circularized. The planet is tidally locked with the same face always pointing towards the star. All other tidal effects are ignored.

(iii) The planet and star are close enough to each other that the rays of starlight reaching the planet are not parallel.

(iv) Particles exist in the atmosphere (or on the surface) across the entire planet, which reflect the starlight.

The flux of the reflected light, $F_{\oplus}$, with frequency $v$ arriving at the distant Earth at time $\mathrm{t}$ is given by

$$
\begin{aligned}
F_{\oplus}(\nu, t)= & \left(\frac{R_{\mathrm{p}}}{d_{\mathrm{p} \oplus}}\right)^{2} \int_{0}^{2 \pi} \int_{0}^{\pi / 2} I_{s \vartheta_{\mathrm{p}} \phi_{\mathrm{p}}}\left(\vartheta_{\mathrm{p}}, \phi_{\mathrm{p}}, \nu, t\right) \\
& \times \sin \vartheta_{\mathrm{p}} \cos \vartheta_{\mathrm{p}} d \vartheta_{\mathrm{p}} d \phi_{\mathrm{p}},
\end{aligned}
$$




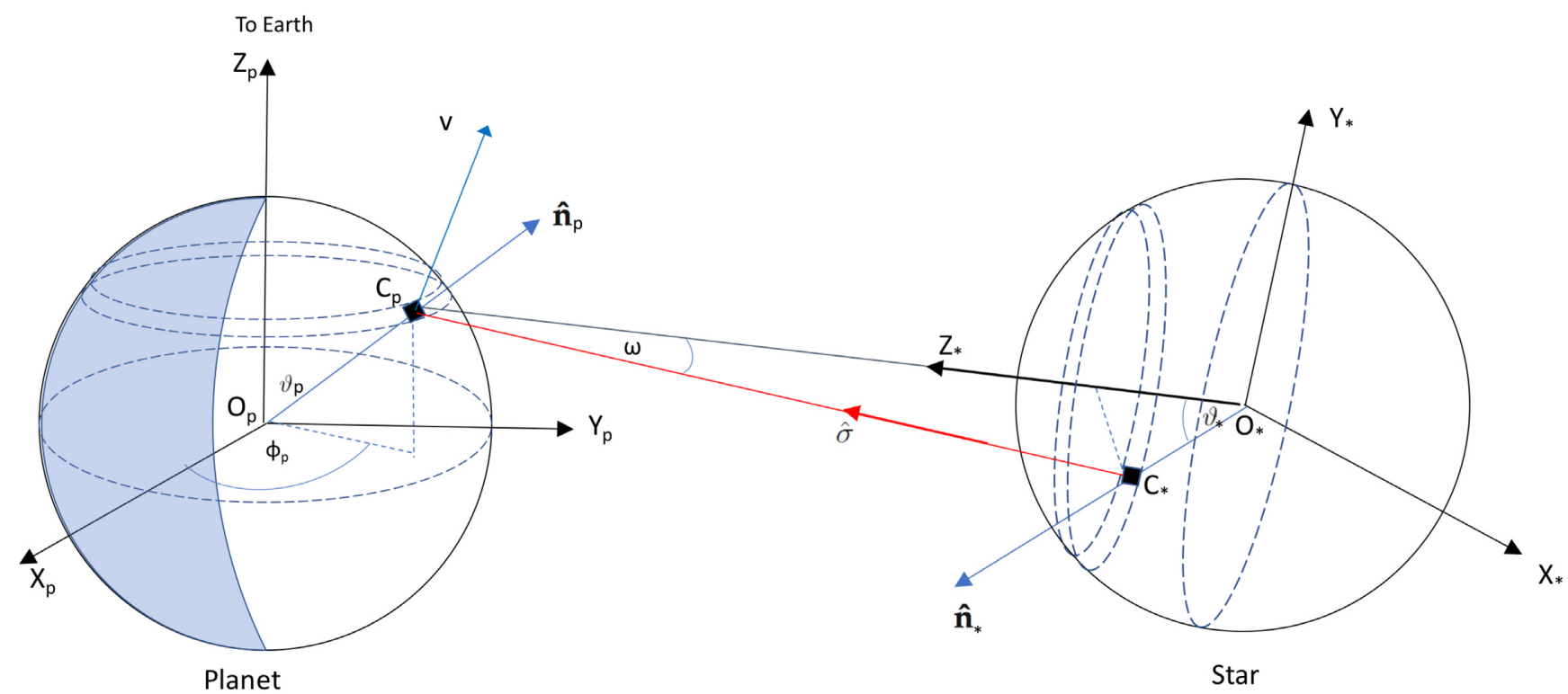

Figure 3. Geometry showing the flux from the star to a point $\left(\vartheta_{p}, \phi_{p}\right)$ on the planet.

where $R_{\mathrm{p}}$ is the radius of the planet, $d_{\mathrm{p} \oplus}$ is the distance from the planet to the Earth, $\vartheta_{\mathrm{p}}, \phi_{\mathrm{p}}$ are colatitude coordinates centred on the planet, and $I_{s \vartheta_{p} \phi_{p}}\left(\vartheta_{p}, \phi_{p}, v, t\right)$ is the intensity of the reflected starlight leaving the surface of the planet at cell $\left(\theta_{\mathrm{p}}, \phi_{\mathrm{p}}\right.$; see Fig. 3$)$. This intensity can be given in terms of the flux $H\left(\vartheta_{\mathrm{p}}, \phi_{\mathrm{p}}, \vartheta_{*}, \phi_{*}, v\right.$ $-\Delta v, t)$ reflecting off the planet cell $\left(\vartheta_{\mathrm{p}}, \phi_{\mathrm{p}}\right)$ from the star cell $\left(\theta_{*}\right.$, $\left.\phi_{*}\right)$ :

$$
\begin{aligned}
I_{s \vartheta_{\mathrm{p}} \phi_{\mathrm{p}}}(v)= & \int_{0}^{2 \pi} \int_{0}^{\pi / 2} \rho\left(\vartheta_{\mathrm{p}}, \phi_{\mathrm{p}}, \vartheta_{*}, \vartheta_{*}\right) \\
& \times H\left(\vartheta_{\mathrm{p}}, \phi_{\mathrm{p}}, \vartheta_{*}, \phi_{*}, v-\Delta \nu, t\right)\left(\hat{\boldsymbol{n}}_{\mathrm{p}} \cdot \hat{\boldsymbol{k}}\right) d \vartheta_{*} \mathrm{~d} \phi_{*},
\end{aligned}
$$

where $\rho\left(\vartheta_{\mathrm{p}}, \phi_{\mathrm{p}}, \vartheta_{*}, \vartheta_{*}\right)$ is the bidirectional reflectance distribution function (ratio of incident flux to reflected intensity), $\hat{\boldsymbol{k}}$ is the unit vector in the direction of the Earth, and the frequency Doppler shift $\Delta v$ is

$\Delta v=v \frac{\Delta \mathrm{v}}{c}$,

where $\Delta \mathrm{v}$ is the velocity shift due to the reflection of the light on the cell $C_{\mathrm{p}}$ and the shift due to the radial motion of the star cell with respect to the planet cell $\Delta v_{r, c_{*} c_{\mathrm{p}}}$. This velocity shift is

$\Delta \mathrm{v}=\mathrm{v}_{\mathbf{p}} \cdot(\hat{\boldsymbol{k}}-\hat{\boldsymbol{\sigma}})+\Delta \mathrm{v}_{r, c_{*} c_{\mathrm{p}}}$.

The surface velocity of the planet cell $\mathbf{v}_{\mathbf{p}}$ is determined in Appendix $\mathrm{B}$ and $\Delta \mathrm{v}_{r, c_{*} c_{\mathrm{p}}}$ is determined in Appendix $\mathrm{C}$.

We determine the flux $F_{\vartheta_{\mathrm{p}} \phi_{\mathrm{p}}}(v)$ at frequency $v$ arriving at a planet cell centred at $\left(\vartheta_{\mathrm{p}}, \phi_{\mathrm{p}}, R_{\mathrm{p}}\right)$ by integrating all the contributions from the star cells

$F_{\vartheta_{\mathrm{p}} \phi_{\mathrm{p}}}(\nu)=\int_{0}^{2 \pi} \int_{0}^{\pi / 2} H\left(\vartheta_{\mathrm{p}}, \phi_{\mathrm{p}}, \vartheta_{*}, \phi_{*}, v-\Delta v_{*}, t\right) \mathrm{d} \vartheta_{*} \mathrm{~d} \phi_{*}$,

where $H\left(\vartheta_{\mathrm{p}}, \phi_{\mathrm{p}}, \vartheta_{*}, \phi_{*}, v, t\right)$ is zero when starlight is blocked from reaching the planet cell:

$H\left(\vartheta_{\mathrm{p}}, \phi_{\mathrm{p}}, \vartheta_{*}, \phi_{*}, v, t\right)=\left\{\begin{array}{ll}0, & \text { if } \hat{\sigma} \cdot \hat{n}_{*}<0 \\ 0, & \text { if }-\hat{\sigma} \cdot \hat{n}_{\mathrm{p}}<0\end{array}\right.$, otherwise non-zero when

$$
\begin{aligned}
& H\left(\vartheta_{\mathrm{p}}, \phi_{\mathrm{p}}, \vartheta_{*}, \phi_{*}, v, t\right)=I_{*}\left(\vartheta_{*}, \phi_{*}, v, t\right)\left(-\hat{n}_{\mathrm{p}} \cdot \hat{\sigma}\right) \\
& \quad \times\left(\frac{-R_{*} \sin \vartheta_{*}}{\sqrt{R_{*}^{2}+d^{2}-2 R_{*} d \cos \vartheta_{*}}}+\frac{R_{*} d \sin \vartheta_{*}\left(d-R_{*} \cos \vartheta_{*}\right)}{\left(R_{*}^{2}+d^{2}-2 d R_{*} \cos \vartheta_{*}\right)^{3 / 2}}\right),
\end{aligned}
$$

and where $\hat{n}_{\mathrm{p}}, \hat{n}_{*}$ are the unit normals to the surface of the planet cell and star cell, $\hat{\sigma}$ is the unit vector in the direction of the planet cell from the star cell, $\mathrm{d}$ is the distance from the planet cell to the centre of the star, and $\Delta \nu_{*}$ is the frequency Doppler shift due to the motion of the star cell. $I_{*}\left(\vartheta_{*}, \phi_{*}, v, t\right)$ is the intensity of light from the surface of the star (the rotationally unbroadened stellar spectrum).

\subsection{RESTART}

RESTART is a software program that produces a synthetic spectrum of reflected light from hot Jupiters on circular orbits based on the spectrum of the star and the orbital parameters of the star-planet system. The code is written in Java and is portable to any system that supports the Java Virtual Machine. RESTART is available on request from the author.

There are two input files: the non-rotationally broadened spectrum or CCF of the star and the parameters file containing the orbital details of the system and other configuration parameters. Table 1 gives an example of the contents of the configuration parameters file. The only output file is the spectrum of the reflected light. The files are in the format of comma separated files (csv files).

RESTART produces the output by performing a numerical integration of equation (35) in order to determine the flux of the reflected starlight observed from the Earth.

\section{RESULTS FROM RUNNING RESTART}

We ran the RESTART software for three systems. The first for a very hot Jupiter using the orbital parameters for WASP-19, the second for $51 \mathrm{Peg}$, and thirdly for an Earth analogue at $10 \mathrm{pc}$. 
Table 1. Input parameters for the very hot Jupiter.

\begin{tabular}{lccl}
\hline Parameter & Value & Units & References \\
\hline$t_{0}$ & 2455168.96801 & BJD & Hellier et al. (2011) \\
$\mathrm{P}$ & 0.78884 & $\mathrm{~d}$ & Hellier et al. (2011) \\
$m_{\mathrm{p}}$ & 1.168 & $m_{\mathrm{Jup}}$ & Hellier et al. (2011) \\
$R_{\mathrm{p}}$ & 1.386 & $R_{\mathrm{Jup}}$ & Hellier et al. (2011) \\
$m_{*}$ & 0.97 & $\mathrm{M}_{\odot}$ & Hellier et al. (2011) \\
$R_{*}$ & 0.99 & $\mathrm{R}_{\odot}$ & Hellier et al. (2011) \\
$\Omega$ & 0 & $\mathrm{deg}$ & \\
$i$ & 79.4 & $\mathrm{deg}$ & Hellier et al. (2011) \\
$\mathrm{a}$ & 0.01655 & $\mathrm{au}$ & Hellier et al. (2011) \\
$d_{* \oplus}$ & 270.409129 & $\mathrm{pc}$ & Gaia Collab. (2018) \\
vsinI & 4.63 & $\mathrm{~km} \mathrm{~s}$ & Hellier et al. (2011) \\
$P_{*, \text { rot }}$ & 10.5 & $\mathrm{~d}$ & Hebb et al. (2010) \\
Resolution & 40 & - & - \\
$\rho$ & $1 / \pi$ & - & - \\
\hline
\end{tabular}

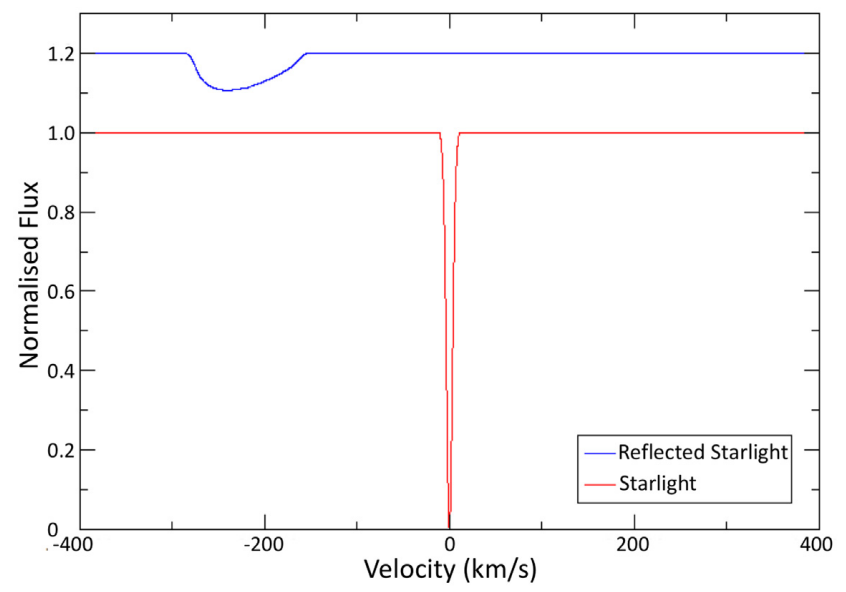

Figure 4. In red the rotationally deconvolved absorption line from WASP19 and in blue the reflected starlight as seen from an observer on the Earth. The reflected starlight has been shifted by 0.2 units.

Finally, we explore the impact on line depth and full width at half-maximum (FWHM) for a hot Jupiter-type system by changing a number of the key parameters of the system in turn: stellar radius, planet radius, stellar rotation, and then planet to star distance.

\subsection{Results for a very hot Jupiter system WASP-19b}

The main input to RESTART is typically the non-rotationally broadened spectrum of the star. This spectrum can be obtained by deconvolving the observed stellar spectrum with a rotational broadening kernel.

In this analysis, we use a simple single synthetic absorption line generated from a Gaussian function with FWHM $=8.963 \mathrm{~km} \mathrm{~s}^{-1}$ - taken from FWHM of HARPS CCFs and deconvolved using the vsinI in Table 1. The CCFs were taken from the HARPS data archive and were based on observations from the $3.6 \mathrm{~m}$ telescope at La SillaParanal Observatory as part of the ESO program 084-C-0185. The line is centred at $0 \mathrm{~km} \mathrm{~s}^{-1}$ as shown in Fig. 4 and has depth 1 relative to the continuum.

The input parameters for the system are given in Table 1 . These parameters include the resolution number of 40 which means that the star and planet surface are broken into $40 \times 2 * 40=3200$ elements for the integration. The bidirectional reflection distribution coefficient was set to a constant $\frac{1}{\pi}$, so there would be isotropic scattering.

We ran RESTART with input time when the planet is at greatest elongation $(t=2455169.16522 d)$. Fig. 4 shows the normalized spectrum of the reflected light as seen from the Earth. The asymmetry in the line is due to only one side of the planet being illuminated as viewed from the Earth and due to the planet observed as rotating from the Earth with period equal to that of the orbital period.

At greatest elongation, we would expect the RV of light reflected from the substellar point of the planet to be at the velocity semiamplitude where

$K_{\text {planet }}=\frac{2 \pi a}{P} \frac{\sin I}{1+m_{\mathrm{p}} / m_{*}}=224 \mathrm{~km} \mathrm{~s}^{-1}$.

We fitted the reflected light to a Gaussian using the LevenbergMarquardt algorithm and obtained an FWHM $=80.3 \mathrm{~km} \mathrm{~s}^{-1}$, which is over 10 times the FWHM of the line from the star. The Gaussian fit gave the centre of the line as $-227.8 \mathrm{~km} \mathrm{~s}^{-1}$ and an amplitude of 0.1 .

We checked the geometric albedo by running RESTART again this time setting the inclination to $90 \mathrm{deg}$ and the date to that corresponding to when the phase angle $\alpha=0$ (time of secondary transit).

The expected geometric albedo can be derived from the following equation where the flux ratio between the reflected light and the light from the star and the planet (Charbonneau et al. 1999; Collier Cameron et al.1999, 2002) is

$\epsilon(\alpha, \lambda)=\frac{F_{E, \mathrm{p}}(\alpha, \lambda)}{F_{E, *}(\lambda)}=\Phi(\alpha, \lambda) p(\lambda) \frac{R_{\mathrm{p}}^{2}}{d_{* \mathrm{p}}^{2}}$,

where $\Phi(\alpha, \lambda)$ is the the orbital phase function describing the amount of light reflected towards the observer at a given phase angle $\alpha$ and wavelength $\lambda, \mathrm{p}(\lambda)$ is the geometric albedo, $R_{\mathrm{p}}$ is the radius of the planet, and $d_{* \mathrm{p}}$ is the distance from the planet to the star. All of the parameters on the right-hand side of the equation except the geometric albedo are in Table 1 and the fluxes ratio on the left-hand side comes from the continuum levels of the input spectrum file and the output spectrum file.

The value obtained was $\mathrm{p}=0.68$, which was only slightly higher than the $2 / 3$ that would be expected for an isotropically scattered Lambert disc.

We next explored the phase curve for the planet by running RESTART for orbital phase $\theta=\{0, \pi / 4, \pi / 2,3 \pi / 2, \pi, 5 \pi / 4,3 \pi / 2\}$, where $\theta=0$ is at time of central transit and having reset the inclination back to $79.4 \mathrm{deg}$. The phase angle $\alpha$ is related to the orbital phase by

$\cos \alpha=-\sin I \cos \theta$,

from Mislis et al. (2012).

We normalized the flux values returned so that the flux at $\alpha=0$ is 1 and plotted them against the curve of the Lambert phase function for this system (Fig. 5). If the planet reflects light isotropically according to Lambert's law and we assume that the scattering phase function is grey - independent of wavelength - then we have from Sobolev (1975) that the Lambert phase function is

$\Phi(\alpha)=\frac{1}{\pi}[\sin \alpha+(\pi-\alpha) \cos \alpha]$.

The figure shows that the reflected light from our model closely follows the Lambert phase function.

Finally, we examined the line formed at time of secondary transit (superior conjunction). Fig. 6 shows the line. It is as expected 


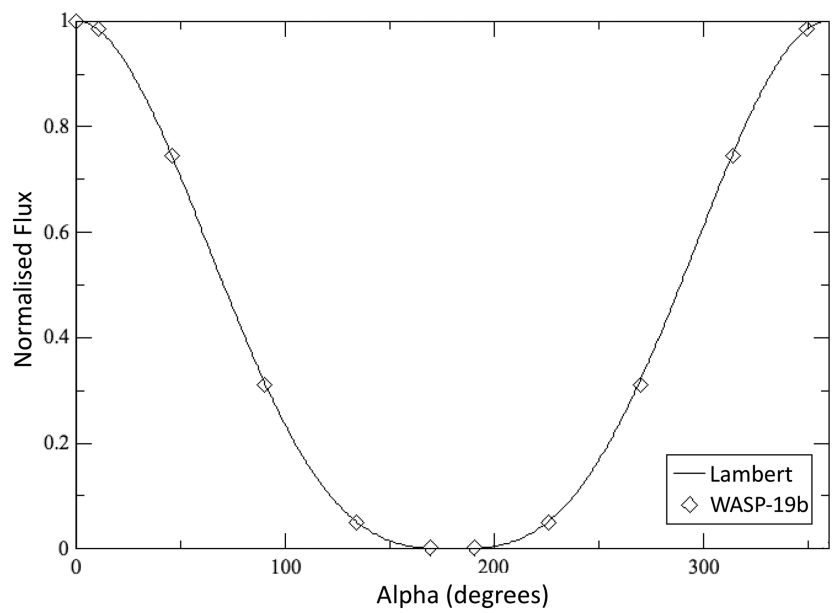

Figure 5. Phase curve for reflected light of WASP-19b along with Lambert Phase Curve for comparison.

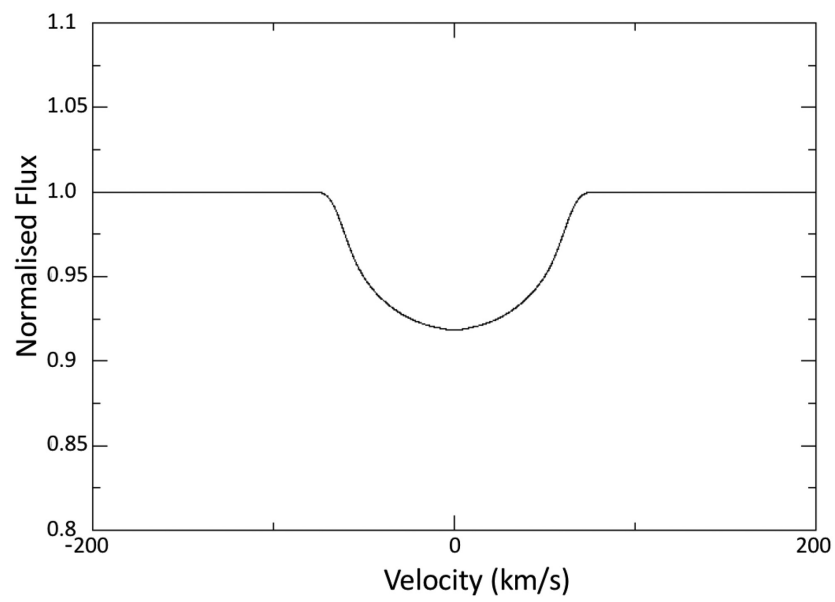

Figure 6. Absorption line for reflected light for a very hot Jupiter at time of secondary transit.

symmetric about velocity $0 \mathrm{~km} \mathrm{~s}^{-1}$ and when fitted with a Gaussian has an FWHM of $90.5 \mathrm{~km} \mathrm{~s}^{-1}$ and a line depth of 0.09 .

\subsection{Results for 51 Peg b system}

As for WASP-19, we use a simple single synthetic absorption line generated from a Gaussian function with FWHM $=7.36 \mathrm{~km} \mathrm{~s}^{-1}$ taken from FWHM of HARPS CCFs and deconvolved using the vsinI in Table 2. The CCFs were taken from the HARPS data archive and were based on observations from the $3.6 \mathrm{~m}$ telescope at La SillaParanal Observatory as part of the ESO program 091.C-0271. The line is centred at $0 \mathrm{~km} \mathrm{~s}^{-1}$ as shown in Fig. 7 and has depth 1 relative to the continuum.

The input parameters for the system are given in Table 2 . We used a planet radius of $1.6 R_{\text {Jup }}$ as this according to the detection paper corresponded to a geometric albedo of $2 / 3$.

We ran RESTART with input time when the planet is at greatest elongation ( $t=2456022.3137 \mathrm{~d}$ ). Fig. 7 shows the normalized spectrum of the reflected light as seen from the Earth. The asymmetry in the line is due to only one side of the planet being illuminated as viewed from the Earth and due to the planet being observed
Table 2. Input parameters for 51 Peg system.

\begin{tabular}{lccl}
\hline Parameter & Value & Units & References \\
\hline$t_{0}$ & 2456021.256 & BJD & Martins et al. (2015) \\
$\mathrm{P}$ & 4.231 & $\mathrm{~d}$ & Martins et al. (2015) \\
$m_{\mathrm{p}}$ & 0.457 & $m_{\text {Jup }}$ & Martins et al. (2015) \\
$R_{\mathrm{p}}$ & 1.6 & $R_{\text {Jup }}$ & Martins et al. (2015) \\
$m_{*}$ & 1.04 & $\mathrm{M}_{\odot}$ & Santos et al. (2003) \\
$R_{*}$ & 1.237 & $\mathrm{R}_{\odot}$ & van Belle \& von Braun (2009) \\
$\Omega$ & 0.0 & $\mathrm{deg}$ & \\
$i$ & 80 & $\mathrm{deg}$ & Martins et al. (2015) \\
$\mathrm{a}$ & 0.052 & $\mathrm{au}$ & Martins et al. (2015) \\
$d_{* \oplus}$ & 14.7 & $\mathrm{pc}$ & Gaia Collab. (2018) \\
vsinI & 2.8 & $\mathrm{~km} \mathrm{~s} \mathrm{~s}^{-1}$ & Mayor \& Queloz (1995) \\
$P_{*, \text { rot }}$ & 37.0 & $\mathrm{~d}$ & Mittag, Schmitt \& Schröder (2018) \\
Resolution & 30 & - & - \\
$\rho$ & $1 / \pi$ & - & - \\
\hline
\end{tabular}

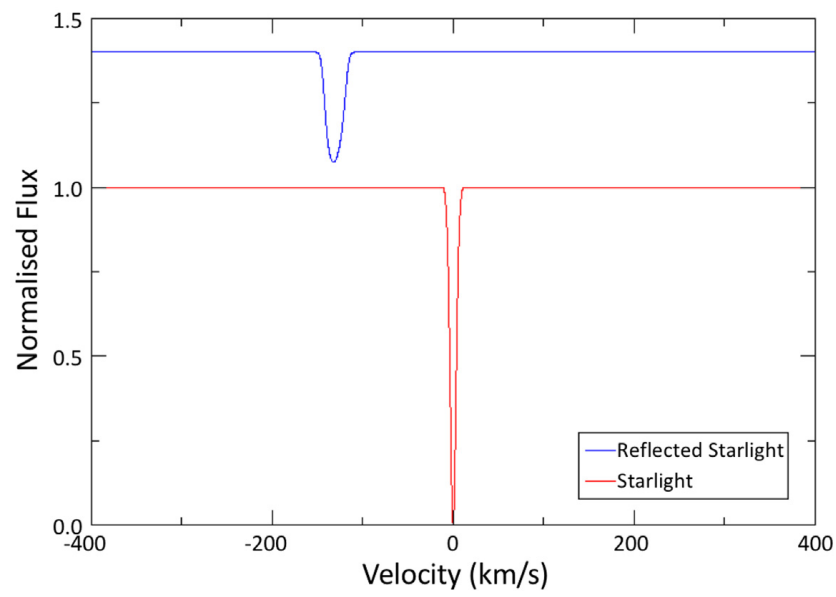

Figure 7. In red the rotationally deconvolved absorption line from $51 \mathrm{Peg}$ and in blue the reflected starlight from 51 Peg b. The reflected starlight has been shifted by 0.4 units.

as rotating from the Earth with period equal to that of the orbital period.

At greatest elongation, we would expect the RV of light reflected from the substellar point of the planet to be at the velocity semiamplitude where

$K_{\text {planet }}=\frac{2 \pi a}{P} \frac{\sin I}{1+m_{\mathrm{p}} / m_{*}}=131 \mathrm{~km} \mathrm{~s}^{-1}$.

We fitted the reflected light to a Gaussian using the LevenbergMarquardt algorithm and obtained an FWHM $=19.0 \mathrm{~km} \mathrm{~s}^{-1}$. The Gaussian fit gave the centre of the line as $-131.2 \mathrm{~km} \mathrm{~s}^{-1}$ and an amplitude of 0.349 .

Fig. 7 shows in detail the reflected light absorption line. The line is slightly asymmetric with the deepest part of the line occurring at a velocity of $-132 \mathrm{~km} \mathrm{~s}^{-1}$. The asymmetry in the line is due to only one side of the rotating planet being illuminated as seen from the Earth.

We examined the phase curve for the planet by running RESTART for a number of phases. We normalized the flux values returned so that the flux at $\alpha=0$ is 1 and plotted them against the curve of the Lambert phase function (Fig. 8). This figure shows that the reflected light from our model closely follows the Lambert phase function.

Finally, we checked the FWHM and amplitude of the line RESTART predicts against the detected signal from Martins et al. 


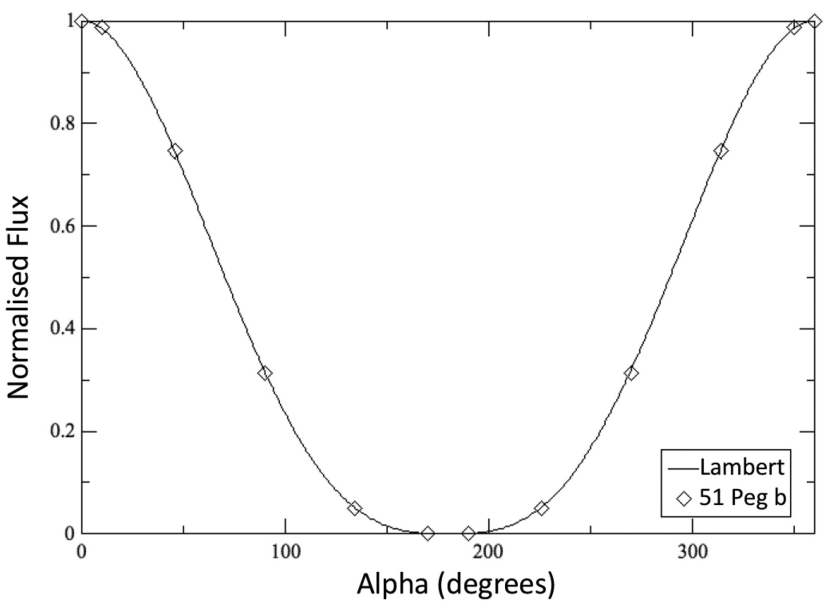

Figure 8. Phase curve for reflected light of $51 \mathrm{Peg} b$ along with Lambert phase curve for comparison.

Table 3. Input parameters for the Earth analogue.

\begin{tabular}{lccc}
\hline Parameter & Value & Units & References \\
\hline$t_{0}$ & 100.0 & BJD & - \\
$\mathrm{P}$ & 365.25 & $\mathrm{~d}$ & - \\
$m_{p}$ & 0.00315 & $m_{\text {Jup }}$ & - \\
$R_{p}$ & 0.08921 & $R_{\text {Jup }}$ & - \\
$m_{*}$ & 1.0 & $\mathrm{M}_{\odot}$ & - \\
$R_{*}$ & 1.0 & $\mathrm{R}_{\odot}$ & - \\
$\Omega$ & 0 & $\mathrm{deg}$ & - \\
$i$ & 90 & $\mathrm{deg}$ & - \\
$\mathrm{a}$ & 1.0 & $\mathrm{au}$ & - \\
$d_{* \oplus}$ & 10 & $\mathrm{pc}$ & - \\
$P_{*, \text { rot }}$ & 24 & $\mathrm{~d}$ & - \\
resolution & 30 & - & - \\
$\rho$ & $1 / \pi$ & - & - \\
\hline
\end{tabular}

(2015) at $\Phi(\alpha)=0.87$. The FWHM from RESTART was $19.6 \mathrm{~km} \mathrm{~s}^{-1}$, which is within $1 \sigma$ of the detected value.

The flux ratio we obtained between the reflected light and the star at the same phase was $1.3 \times 10^{-4}$. However, this is only the fractional difference in the level of the continuum but taking into account the reduction of the line amplitude due to the broadening (line amplitude was 0.41 ) we have a signal level of $5.4 \times 10^{-5}$, which is within $1.5 \sigma$ of the detection result reported by Martins et al. (2015). Increasing the radius of the planet to $1.7 R_{\text {Jup }}$ would bring the flux ratio to the detected value of $6.0 \times 10^{-5}$.

\subsection{Results for a tidally locked Earth analogue}

We used the absorption line generated for 51 Peg as the input stellar spectrum as $51 \mathrm{Peg}$ has a similar spectral type to the Sun. The input parameters for the system are given in Table 3 .

We ran RESTART with input time when the planet is at greatest elongation. Fig. 9 Tab (b) shows the normalized spectrum of the observed reflected light.

At greatest elongation, we would expect the RV of light reflected from the substellar point of the planet to be at the velocity semiamplitude where

$K_{\text {planet }}=\frac{2 \pi a}{P} \frac{\sin I}{1+m_{\mathrm{p}} / m_{*}}=29.8 \mathrm{~km} \mathrm{~s}^{-1}$.
Fitting a Gaussian using the Levenberg-Marquardt algorithm to the line gave its centre at $-29.8 \mathrm{~km} \mathrm{~s}^{-1}$ as expected. Its FWHM of $6.8 \mathrm{~km} \mathrm{~s}^{-1}$ and depth of 0.93 showed broadening compared to the FWHM of $6.4 \mathrm{~km} \mathrm{~s}^{-1}$ and depth of 1.0 of the line before it was reflected. This is again due to light originating from opposing limbs of the star having different angles of incidence on the planet. In order to confirm this, we reduced the radius of the Sun by a factor of 10 and reran RESTART. This resulted in a line of depth 0.99 and FWHM of $6.4 \mathrm{~km} \mathrm{~s}^{-1}$, which were within 1 percent of the figures for the original line.

We checked the geometric albedo by running RESTART again this time setting the date to that corresponding to when the phase angle $\alpha=0$ (time of secondary transit). The value for the geometric albedo obtained was $p=0.668$, which was consistent with the $2 / 3$ that would be expected for a Lambert disc.

We next explored the phase curve for the planet by running RESTART for orbital phase $\theta=\{0, \pi / 4, \pi / 2,3 \pi / 2, \pi, 5 \pi / 4,3 \pi / 2\}$, where $\theta=0$ is at time of centre of primary transit.

We normalized the flux values returned so that the flux at $\alpha=0$ is 1 and plotted them against the curve of the Lambert phase function (Fig. 10). This figure shows that the reflected light from our model follows the Lambert phase function.

\subsection{Impact of system parameter changes to line depth and FWHM}

Here, we report on the impact on line depth and FWHM for a hot Jupiter-type system by changing a number of the parameters of the system including star radius, planet radius, planet-to-star distance, and stellar rotation in turn.

We use the parameters based on those for 51 Peg b as the base for the system except that we set the inclination to $90 \mathrm{deg}$ and the time of observation to be when the planet is fully illuminated with respect to the observer. This permits us to look at the system when the broadened line is symmetric. The parameters are specified in Table 4

The line depths and FWHM for the broadened lines output from RESTART are determined by fitting a Gaussian using the LevenbergMarquardt algorithm as before.

The stellar radius was varied between 0.1 and 3 solar radii covering the domain of main-sequence stars from $\mathrm{M}$ dwarfs to $\mathrm{A}$ stars. The results are shown in Fig. 11. The FWHM is correlated with the stellar radius. We can also see that from approximately $0.5 R_{\odot}$ upwards in stellar radius the increase in FWHM is approximately linear. In terms of line amplitude against stellar radius, below $0.5 R_{\odot}$ the relationship is linear as it heads asymptotically towards the level of no broadening and a line amplitude of 1 .

Next, the planet radius was varied between 0.09 and 13 Jupiter radii covering the domain of the Earth-sized planets up towards the largest planets which approach 2 Jupiter radii and then beyond that in order to show the asymptotic relationship. The results are shown in Fig. 12. The FWHM of the spectral line for the planet calculated from RESTART increases as we increase the planet radius. In particular, this relationship when the planet radius is large $(>8$ Jupiter radii) is close to linear. As the planet radius decreases the FWHM asymptotically approaches a value of just over $19 \mathrm{~km} \mathrm{~s}^{-1}$, where the broadening is primarily due to the size of the star.

The line amplitude is inversely correlated with the planet radius. At planet radius 5-13 Jupiter radii the relationship with line amplitude is close to linear and as the planet radius decreases towards zero the line amplitude asymptotically approaches a value of just over 0.41 . 

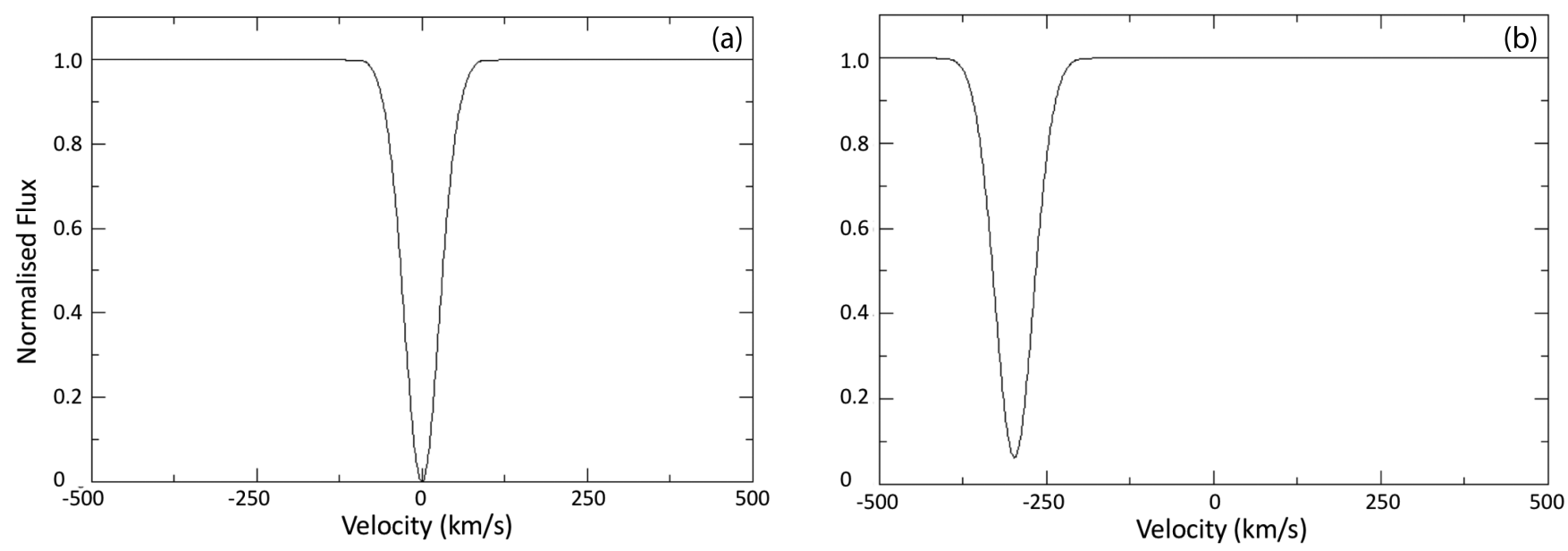

Figure 9. Tab (a) shows the rotationally deconvolved absorption line representing s solar line. Tab (b) shows the reflected light as seen from the Earth. Both tabs have had the continuum normalized to 1 .

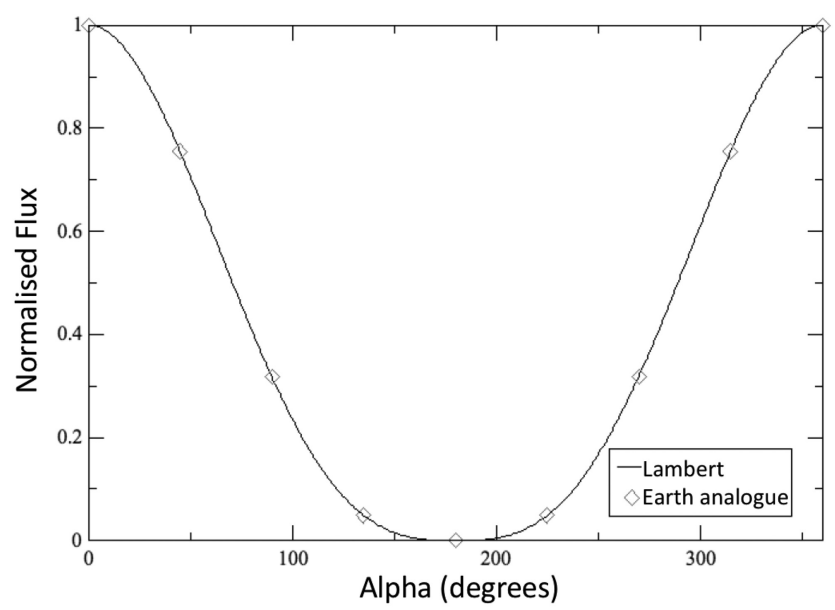

Figure 10. Phase curve for reflected light from the Earth analogue along with Lambert phase curve for comparison.

Table 4. Parameters set for hot Jupiter type system based on 51 Peg used for exploring the impact of system parameter changes to line depth and FWHM.

\begin{tabular}{lccl}
\hline Parameter & Value & Units & References \\
\hline$t_{0}$ & 56020.756 & MJD & Martins et al. (2015) \\
$\mathrm{P}$ & 4.231 & $\mathrm{~d}$ & Martins et al. (2015) \\
$m_{\mathrm{p}}$ & 0.457 & $m_{\text {Jup }}$ & Martins et al. (2015) \\
$R_{\mathrm{p}}$ & 1.6 & $R_{\text {Jup }}$ & Martins et al. (2015) \\
$m_{*}$ & 1.04 & $\mathrm{M}_{\odot}$ & Santos et al. (2003) \\
$R_{*}$ & 1.237 & $\mathrm{R}_{\odot}$ & van Belle \& von Braun (2009) \\
$\Omega$ & 0.0 & $\mathrm{deg}$ & - \\
$i$ & 90 & $\mathrm{deg}$ & - \\
$\mathrm{a}$ & 0.052 & $\mathrm{au}$ & Martins et al. (2015) \\
$d_{* \oplus}$ & 14.7 & $\mathrm{pc}$ & Gaia Collab. (2018) \\
vsinI & 2.8 & $\mathrm{~km} \mathrm{~s}^{-1}$ & Mayor \& Queloz (1995) \\
$P_{*, \text { rot }}$ & 37.0 & $\mathrm{~d}$ & Mittag et al. (2018) \\
resolution & 30 & - & - \\
$\rho$ & $1 / \pi$ & - & - \\
FWHM & 7.36 & $\mathrm{~km} \mathrm{~s}^{-1}$ & - \\
\hline & & &
\end{tabular}

The observed period of rotation of the $\operatorname{star}\left(P_{* \text {, rot }}\right)$ was varied between 0.1 and $100 \mathrm{~d}$ next. The results are shown in Fig. 13. As expected the minimum FWHM and maximum line amplitude occurs when the planet and star are in synchronous orbit with each other, which is when the observed stellar rotation period is at the planet orbital period of $4.231 \mathrm{~d}$. As the observed stellar rotation period decreases below the orbital period then the broadening increases and the line amplitude decreases rapidly. For increasing stellar rotation period (as the star spins down), the broadening increases but then flattens out to an FWHM of just over $20 \mathrm{~km} \mathrm{~s}^{-1}$.

Next, we vary the distance between the star and the planet. In doing this, we also modify the orbital period so that the system is consistent with Kepler's laws and we modify the observed stellar rotation so that it is always $30 \mathrm{~d}$ longer than the orbital period of the system. The results are shown in Fig. 14. Here, we see that the broadening is small with FWHM $<10 \mathrm{~km} \mathrm{~s}^{-1}$ until the distance between planet and star is less than 0.25 au and then as we get closer to the star the level of broadening increases and the line amplitude decreases very rapidly.

Finally, we vary the distance between the star and the planet again but this time we also vary the observed stellar rotation, so that its rate is that of the orbital period of the planet and thus the orbits are synchronized. The results are shown in Fig. 15. The broadening is minimal until 0.05 au where the broadening increases steeply with decreasing distance between planet and star. The broadening is due to the large range of incident angles for starlight being reflected from the planet due to the proximity of the star to the planet.

\section{DISCUSSION}

In the development of the model for reflected starlight, we have deliberately excluded a number of mainly lower order effects from the model. Here, we describe some of them and detail how future versions of the model could include them.

We have only including isotropic scattering $\rho=$ constant in our model calculations resulting in the planet phase curves closely resembling those for a Lambert phase curve. For non-isotropic and multiple scattering of reflected light from exoplanets (Seager, Whitney \& Sasselov 2000), we would need to identify types and sizes of scatterers in the atmosphere of the planet along with their relevant phase functions. Models for these scatterers already 

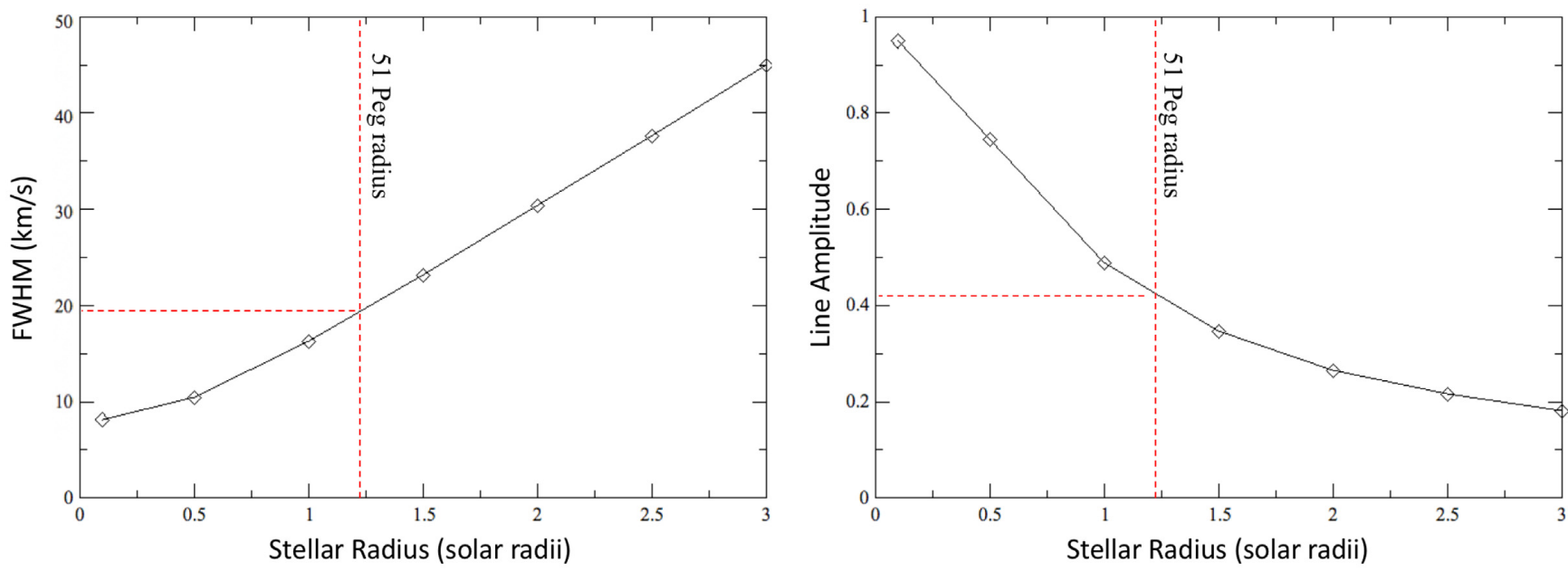

Figure 11. Impact of change of stellar radius on FWHM (left) and line amplitude (right) for a hot Jupiter-type system based on the 51 Peg system. The input parameters for the system are specified in Table 4.
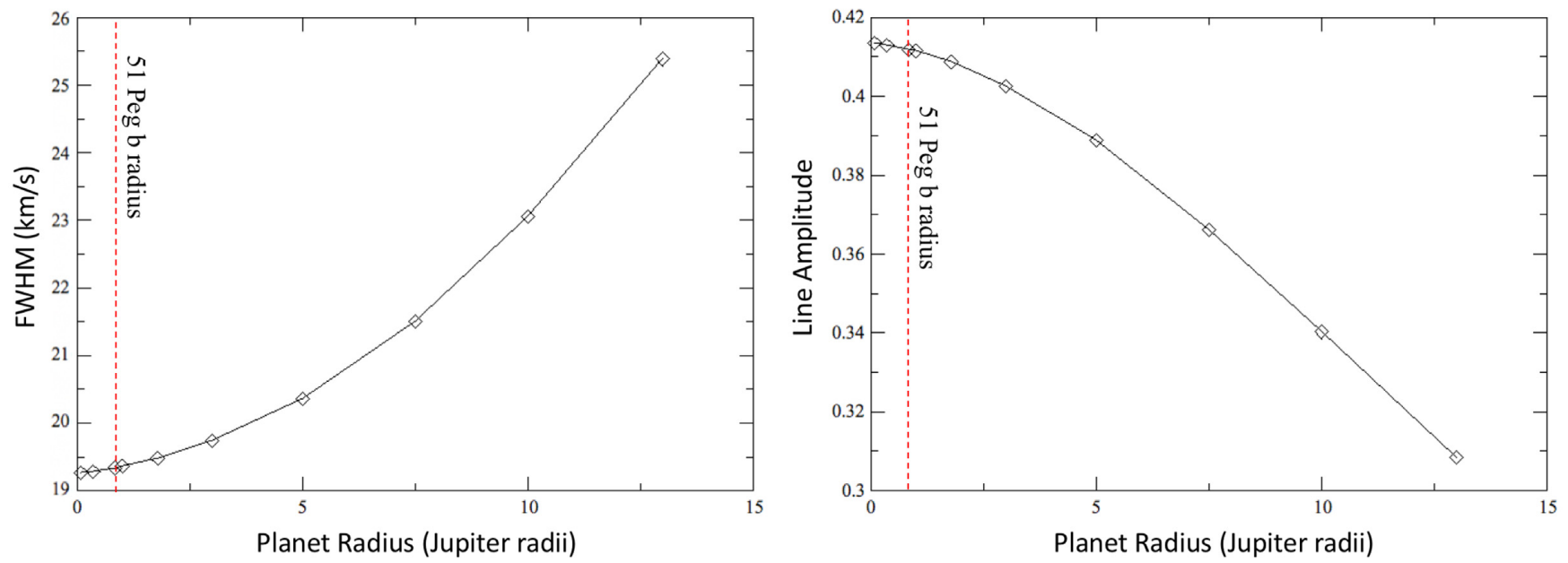

Figure 12. Exoplanet system as for Fig. 11 except showing the impact of change of planet radius on FWHM (left) and line amplitude (right).
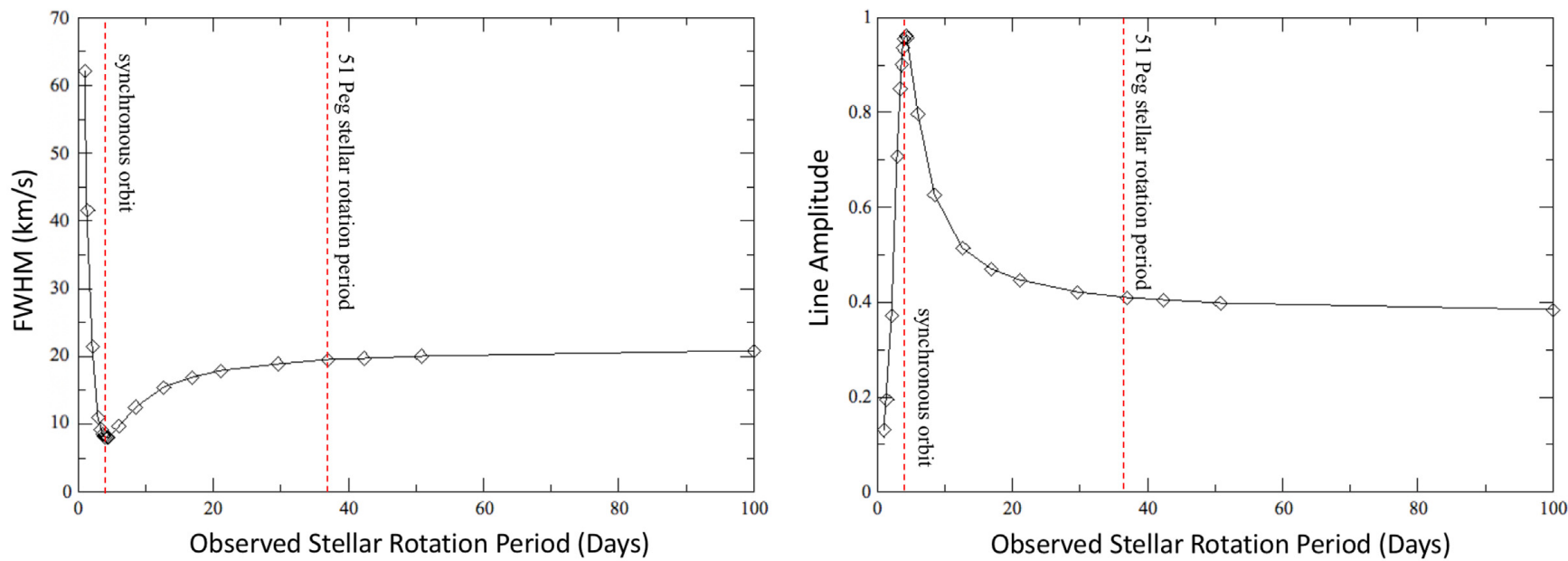

Figure 13. Exoplanet system as for Fig. 11 except showing the impact of change of stellar rotation on FWHM (left) and line amplitude (right). 

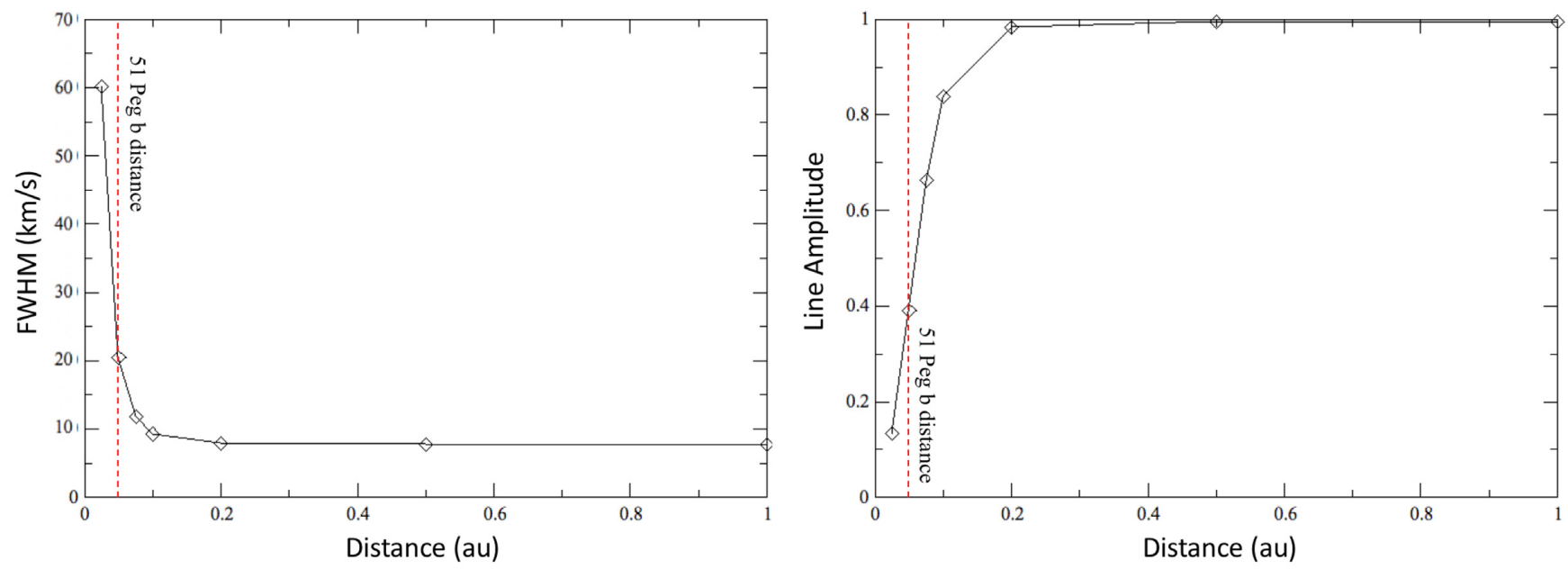

Figure 14. Exoplanet system as for Fig. 11 except showing the impact of change of distance between planet and star on FWHM (left) and line amplitude (right).
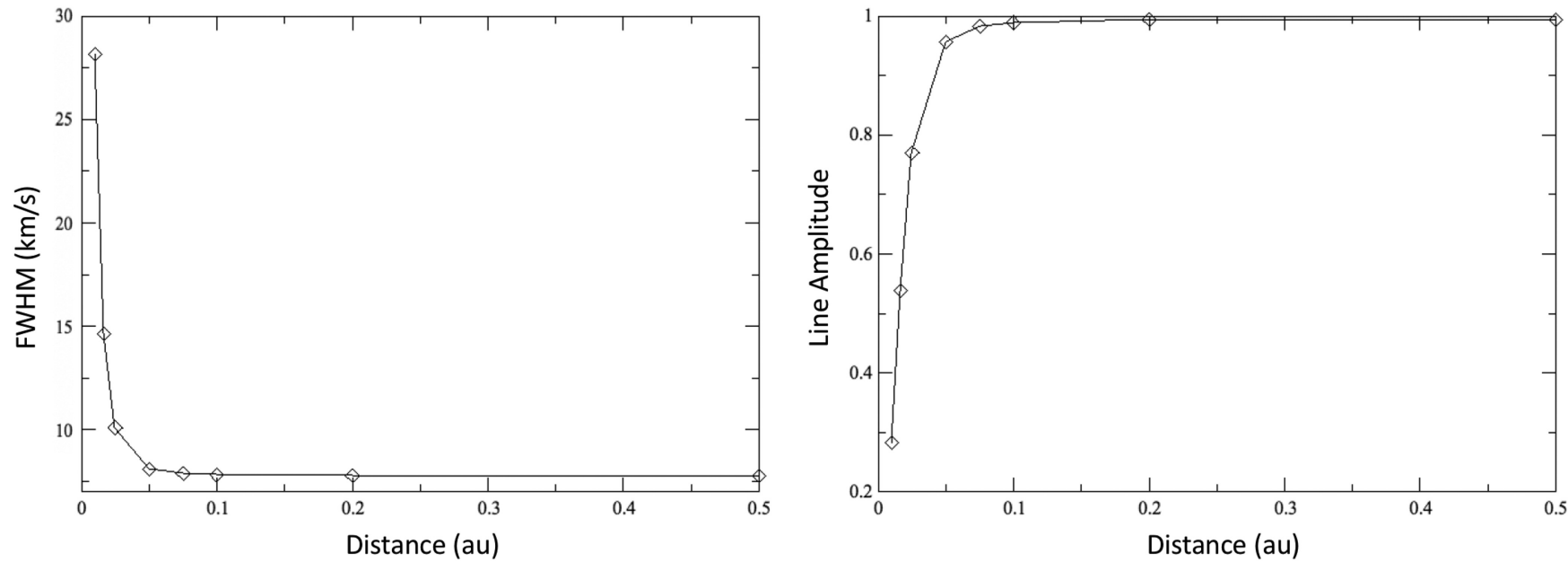

Figure 15. Exoplanet system as for Fig. 11 except showing the impact of change of distance between planet and star on FWHM (left) and Line Amplitude (right) for a synchronized hot Jupiter system.

exist, for example, see the aforementioned paper and could be incorporated into the model.

We have also not taken account of the velocities of the particles/winds in particular those due to recirculation from day to night side, which can be important in particular for hot Jupiters. These winds have been measured (e.g. Louden \& Wheatley 2015) and are typically of the order of a few $\mathrm{km} \mathrm{s}^{-1}$.

In terms of tides, we have not taken account of the deformed shapes of the planet and star in our model. For instance, Correia (2014) estimated the level of deformation of close in exoplanets assuming the deformed shape was in the form of a triaxial ellipsoid. For WASP-19b, they estimated the length of the three axis to be $17.3,15.4$, and $14.8 R_{\oplus}$ under the assumption that if the planet were spherical its radius would be $15.2 R_{\oplus}$. In terms of our model, this means that we would underestimate the flux and line broadening at greatest elongation and overestimate it at time of secondary transit. The size of the deformations is based on assumptions on the Love number used and to date no evidence of the deformations has been observed. We could similarly update the model to include the ellipticity of the star and gravitational darkening due to tides using a model such as that outlined in Jackson et al. (2012). For
WASP-19b, the maximum increase in radius due to tides would be $3.32 \times 10^{-4} R_{*}$.

Although it is expected that most hot Jupiters orbit their parent star in circular orbits and are expected to be tidally locked, we could also lift these assumptions from our model.

\section{CONCLUSIONS}

In this work, we have shown using a simple model of scattering that the Doppler shift of reflected light from planets whose parent star is at a distance where the light rays arriving at the planet can be considered to be parallel, have a different Doppler shift to that of light emitted directly from the planet. The magnitude of this difference in Doppler shift is equivalent to the radial motion of the planet with respect to the star.

We also showed that in the case of planets close to the parent star and in particular hot Jupiters the reflected starlight observed should be broadened due to the difference in angles of incidence of starlight arriving at the planet from different limbs of the star. We gave estimates for this broadening and developed a model that describes 
the scattering that takes place for tidally locked hot Jupiters in circular orbits.

We implemented the model in software (RESTART) and then ran this software tool for three exoplanets: We found that significant broadening occurred for the WASP-19b very hot Jupiter system and significant broadening also took place for the 51 Peg b system. Our results for $51 \mathrm{Peg}$ were consistent with the detection results provided we assumed the planet had size of $1.7 R_{\text {Jup }}$. Very minimal broadening took place for our Earth analogue.

Light curves produced for all three planets showed no significant deviations from a Lambertian phase curve as was expected for isotropic scattering.

\section{ACKNOWLEDGEMENTS}

JS acknowledges support of studentship funded by Queen Mary University of London. This research was also supported by STFC (Science and Technology Facilities Council) Consolidated Grant ST/P000592/1. The authors acknowledge the helpful and constructive comments from the referee. The authors are also grateful to the HARPS team at Observatoire de Genève, Observatoire de Haute-Provence, Laboratoire d'Astrophysique de Marseille, Service d'Aéronomie du CNRS, Physikalisches Institut de Universität Bern, ESO La Silla, and ESO Garching, who built and maintained the HARPS instrument, and were generous enough to make the data public.

\section{REFERENCES}

Anglada-Escudé G., Klioner S. A., Soffel M., Torra J., 2007, A\&A, 462, 371

Borra E. F., Deschatelets D., 2018, MNRAS, 481, 4841

Borucki W. J. et al., 2009, Science, 325, 709

Charbonneau D., Noyes R. W., Korzennik S. G., Nisenson P., Jha S., Vogt S. S., Kibrick R. I., 1999, ApJ, 522, L145

Charrett T. O. H., James S. W., Tatam R. P., 2012, Meas. Sci. Tech., 23, 032001

Claudi R., 2016, Direct Imaging of Faint Companions. Springer International Publishing, Cham, Switzerland, p. 183

Collier Cameron A., Horne K., Penny A., James D., 1999, Nature, 402, 751

Collier Cameron A., Horne K., Penny A., Leigh C., 2002, MNRAS, 330, 187

Correia A. C. M., 2014, A\&A, 570, L5

Gaia Collaboration, 2018, A\&A, 616, A1

Harrington J., Hansen B. M., Luszcz S. H., Seager S., Deming D., Menou K., Cho J. Y.-K., Richardson L. J., 2006, Science, 314, 623

Hebb L. et al., 2010, ApJ, 708, 224

Hellier C., Anderson D. R., Collier-Cameron A., Miller G. R. M., Queloz D., Smalley B., Southworth J., Triaud A. H. M. J., 2011, ApJ, 730, L31

Jackson B. K., Lewis N. K., Barnes J. W., Drake Deming L., Showman A. P., Fortney J. J., 2012, ApJ, 751, 112

Louden T., Wheatley P. J., 2015, ApJ, 814, L24

Martins J. H. C. et al., 2015, A\&A, 576, A134

Martins J. H. C., Santos N. C., Figueira P., Melo C., 2016, Orig. Life Evol. Biosph., 46, 487

Mayor M., Queloz D., 1995, Nature, 378, 355

Mislis D., Heller R., Schmitt J. H. M. M., Hodgkin S., 2012, A\&A, 538, A4

Mittag M., Schmitt J. H. M. M., Schröder K.-P., 2018, A\&A, 618, A48

Murray C. D., Correia A. C. M., 2010, Keplerian Orbits and Dynamics of Exoplanets, University of Arizona Press, Tucson, Arizona, p. 15

Perryman M., 2018, The Exoplanet Handbook. Cambridge Univ. Press, Cambridge

Santos N. C., Israelian G., Mayor M., Rebolo R., Udry S., 2003, A\&A, 398, 363

Schneider J., Dedieu C., Le Sidaner P., Savalle R., Zolotukhin I., 2011, A\&A, 532, A79
Seager S., 2010, Exoplanet Atmospheres: Physical Processes. Princeton Series in Astrophysics. Princeton Univ. Press, Princeton, NJ

Seager S., Sasselov D. D., 1998, ApJ, 502, L157

Seager S., Whitney B. A., Sasselov D. D., 2000, ApJ, 540, 504

Sobolev V., 1975, Light Scattering in Planetary Atmospheres. International Series of Monographs in Natural Philosophy. Pergamon Press, Oxford, United Kingdom

van Belle G. T., von Braun K., 2009, ApJ, 694, 1085

Walker G. A. H. et al., 2006, in Arnold L., Bouchy F., Moutou C., eds, Tenth Anniversary of 51 Peg-b: Status of and Prospects for Hot Jupiter Studies. Observatoire de Haute Provence, France. p. 267

Yeh Y., Cummins H. Z., 1964, Appl. Phys. Lett., 4, 176

\section{APPENDIX A: FLUX AND DOPPLER SHIFT OF REFLECTED LIGHT FROM HOT JUPITERS}

Here, we determine the flux and Doppler shift of the reflected light arriving at the Earth from a tidally locked hot Jupiter in a circular orbit. The geometry we use to determine this is shown in Fig. 3.

We use two main coordinate systems. The first is the Cartesian coordinate system $X_{\mathrm{p}} Y_{\mathrm{p}} Z_{\mathrm{P}}$ centred on the planet, where the $Z_{\mathrm{P}}$ axis is pointing towards the Earth and the $X_{\mathrm{p}}$ and $Y_{\mathrm{p}}$ axis in the plane of the sky as viewed from the Earth. We use a colatitude coordinate system $\left(\vartheta_{\mathrm{p}}, \phi_{\mathrm{p}}, R_{\mathrm{p}}\right)$ to describe the location of points on the surface of the planet.

In order to determine the reflected starlight arriving at the Earth, we first determine the starlight incident at each point $\left(\vartheta_{\mathrm{p}}, \phi_{\mathrm{p}}\right)$ on the surface of the planet.

For this, we use a second stellar Cartesian coordinate system $X_{*} Y_{*} Z_{*}$ centred on the star and that has $Z_{*}$ axis pointing towards $\left(\vartheta_{\mathrm{p}}, \phi_{\mathrm{p}}\right)$ as shown in Fig. 3 . This stellar coordinate system also has its associated colatitude system $\left(\vartheta_{*}, \phi_{*}, R_{*}\right)$ in order to describe points on the surface of the star. Let $\mathrm{d}$ be the distance between $C_{\mathrm{P}}$ and the centre of the star. $C_{\mathrm{P}}$ is the point $\left(R_{\mathrm{p}} \cos \phi_{\mathrm{p}} \sin \vartheta_{\mathrm{p}}, R_{\mathrm{p}} \sin \phi_{\mathrm{p}} \sin \vartheta_{\mathrm{p}}\right.$, $\left.R_{\mathrm{p}} \cos \vartheta_{\mathrm{p}}\right)$ in the planet frame. In this frame, we assume we have that $O_{*}=\left(x_{*}, y_{*}, z_{*}\right)$ and we can determine d as follows:

Angle $C_{\mathrm{p}} \hat{O}_{\mathrm{p}} O_{*}=\gamma$ is

$\cos (\gamma)=\cos \phi_{\mathrm{p}} \sin \vartheta_{\mathrm{p}} \frac{x_{*}}{a}+\sin \phi_{\mathrm{p}} \sin \vartheta_{\mathrm{p}} \frac{y_{*}}{a}+\cos \vartheta_{\mathrm{p}} \frac{z_{*}}{a}$.

Using the cosine rule, we have

$d^{2}=a^{2}+R_{\mathrm{p}}^{2}-2 a R_{\mathrm{p}} \cos \gamma$,

and we can now determine $\tan \omega$ as

$\tan \omega=\frac{R_{*} \sin \vartheta_{*}}{d-R_{*} \cos \vartheta_{*}}$.

$\sin \omega$ and $\cos \omega$ and their implicit differentials are

$$
\begin{gathered}
\sin \omega=\frac{R_{*} \sin \vartheta_{*}}{\sqrt{R_{*}^{2}+d^{2}-2 R_{*} d \cos \vartheta_{*}}}, \\
\cos \omega \mathrm{d} w=\left(\frac{R_{*} \cos \vartheta_{*}}{\sqrt{R_{*}^{2}+d^{2}-2 d R_{*} \cos \vartheta_{*}}}\right. \\
\left.-\frac{R_{*}^{2} d \sin ^{2} \vartheta_{*}}{\left(R_{*}^{2}+d^{2}-2 d R_{*} \cos \vartheta\right)^{3 / 2}}\right) \mathrm{d} \vartheta_{*}, \\
\cos \omega=\frac{d-R_{*} \cos \vartheta_{*}}{\sqrt{R_{*}^{2}+d^{2}-2 R_{*} d \cos \vartheta_{*}}}, \\
+\frac{-R_{*} \sin \vartheta_{*}}{\sqrt{R_{*}^{2}+d^{2}-2 R_{*} d \cos \vartheta_{*}}} \\
+\frac{R_{*} d \sin \vartheta_{*}\left(d-R_{*} \cos \vartheta_{*}\right)}{\left(R_{*}^{2}+d^{2}-2 d R_{*} \cos \vartheta\right)^{3 / 2}} \mathrm{~d} \vartheta_{*} .
\end{gathered}
$$


The solid angle of the star subtended by the planet cell is

$\Omega_{\theta_{\mathrm{p}} \phi_{\mathrm{p}}}=\int_{0}^{2 \pi} \int_{0}^{\tan ^{-1}\left(\frac{R_{*}}{d}\right)} \sin \omega \mathrm{d} \omega \mathrm{d} \phi_{*}-$ blocked portion.

The blocked portion of the solid angle is the portion where light cannot travel to the planet cell due to some part of the star or planet being in its path. In order to take account of this, when we calculate the integral numerically for each element we check whether

$0 \leq \hat{\sigma} \cdot \hat{n}_{*}<1$

and

$0 \leq-\hat{\sigma} \cdot \hat{n}_{\mathrm{p}}<1$.

If the light is blocked, then at least one of the two inequalities will not hold and instead of using the $\sin \omega$ factor in the integral we use 0 instead.

The flux at the point $\left(\vartheta_{\mathrm{p}}, \phi_{\mathrm{p}}\right)$ of the planet from the star is

$$
\begin{aligned}
F_{\vartheta_{\mathrm{p}} \phi_{\mathrm{p}}}(\nu)= & \int_{0}^{2 \pi} \int_{0}^{\tan ^{-1}\left(\frac{R_{*}}{d}\right)} I_{*}\left(\vartheta_{*}, \phi_{*}, v-v_{*}, t\right) \\
& \times\left(-\hat{n}_{\mathrm{p}} \cdot \sigma\right) \sin \omega \mathrm{d} \omega \mathrm{d} \phi_{*},
\end{aligned}
$$

where $v_{*}$ is the frequency Doppler shift due to the motion of the star cell.

We have to work out the dot product $\left(\hat{n}_{\mathrm{p}} \cdot \sigma\right)$, which is complicated by the fact that currently we only know the position of the cell $C_{*}$ in the coordinates of the $\operatorname{star}\left(R_{*} \cos \phi_{*} \sin \vartheta_{*}, R_{*} \sin \phi_{*} \sin \vartheta_{*}\right.$, $\left.R_{*} \cos \vartheta_{*}\right)_{*}$.

In order to move to the planet frame from the star frame, we have two rotations in order to align the $Z_{*}$ axis and a translation to perform (see Fig. A1).

In terms of vectors, we have

$\overrightarrow{O_{\mathrm{p}} C_{*}}=\overrightarrow{O_{\mathrm{p}} O_{*}}+\overrightarrow{O_{*} C_{*}}=\overrightarrow{O_{*} C_{*}}+\boldsymbol{R}_{x}\left(-\Lambda_{x}\right) \boldsymbol{R}_{y}\left(-\Lambda_{y}\right) \overrightarrow{O_{*} C_{*}^{\prime}}$,

where $\boldsymbol{R}_{x}$ and $\boldsymbol{R}_{y}$ are rotation matrices about the $X$ - and $Y$-axes, respectively, and the angles $\Lambda_{x}$ and $\Lambda_{y}$ are the angles so that the rotations $\boldsymbol{R}_{y}\left(\Lambda_{y}\right) \boldsymbol{R}_{x}\left(\Lambda_{x}\right)$ move the $Z_{\mathrm{p}}$ axis to be in the same direction as the $Z_{*}$ axis.

In component form, we have

$\left[\begin{array}{c}C_{* x} \\ C_{* y} \\ C_{* z}\end{array}\right]_{p}=\left[\boldsymbol{R}_{x}\left(-\Lambda_{x}\right) \boldsymbol{R}_{y}\left(-\Lambda_{y}\right)\right]\left[\begin{array}{c}R_{*} \cos \phi_{*} \sin \vartheta_{*} \\ R_{*} \sin \phi_{*} \sin \vartheta_{*} \\ R_{*} \cos \vartheta_{*}\end{array}\right]_{*}+\left[\begin{array}{c}x_{*} \\ y_{*} \\ z_{*}\end{array}\right]_{p}$.

The subscripts on the matrix correspond to the Cartesian coordinate frame the components are in ( $\mathrm{p}$ for planet and ${ }^{*}$ for star)

Expanding out the rotation matrices, we have

$$
\begin{aligned}
{\left[\begin{array}{c}
C_{* x} \\
C_{* y} \\
C_{* z}
\end{array}\right]_{p} } & {\left[\begin{array}{ccc}
\cos \Lambda_{y} & 0 & -\sin \Lambda_{y} \\
\sin \Lambda_{x} \sin \Lambda_{y} & \cos \Lambda_{x} & \sin \Lambda_{x} \cos \Lambda_{y} \\
\cos \Lambda_{x} \sin \Lambda_{y} & -\sin \Lambda_{x} & \cos \Lambda_{x} \cos \Lambda_{y}
\end{array}\right] } \\
& \times\left[\begin{array}{c}
R_{*} \cos \phi_{*} \sin \vartheta_{*} \\
R_{*} \sin \phi_{*} \sin \vartheta_{*} \\
R_{*} \cos \vartheta_{*}
\end{array}\right]_{*}+\left[\begin{array}{c}
x_{*} \\
y_{*} \\
z_{*}
\end{array}\right]_{p} .
\end{aligned}
$$

Expanding out the components, we have

$$
\begin{aligned}
C_{* x}= & \cos \Lambda_{y} R_{*} \cos \phi_{*} \sin \vartheta_{*}-\sin \Lambda_{y} R_{*} \cos \vartheta_{*}+x_{*}, \\
C_{* y}= & \sin \Lambda_{x} \sin \Lambda_{y} R_{*} \cos \phi_{*} \sin \vartheta_{*}+\cos \Lambda_{x} R_{*} \sin \phi_{*} \sin \vartheta_{*} \\
& +\sin \Lambda_{x} \cos \Lambda_{y} R_{*} \cos \vartheta_{*}+y_{*}, \\
C_{* z}= & \cos \Lambda_{x} \sin \Lambda_{y} R_{*} \cos \phi_{*} \sin \vartheta_{*}-\sin \Lambda_{x} R_{*} \sin \phi_{*} \sin \vartheta_{*} \\
& +\cos \Lambda_{x} \cos \Lambda_{y} R_{*} \cos \vartheta_{*}+z_{*} .
\end{aligned}
$$

We determine the components of $\sigma=\left(\sigma_{x}, \sigma_{y}, \sigma_{z}\right)^{T}$ as

$$
\begin{aligned}
\sigma_{x}= & R_{p} \cos \phi_{p} \sin \vartheta_{p} \\
& -\left(\cos \Lambda_{y} R_{*} \cos \phi_{*} \sin \vartheta_{*}-\sin \Lambda_{y} R_{*} \cos \vartheta_{*}+x_{*}\right), \\
\sigma_{y}= & R_{p} \sin \phi_{*} \sin \vartheta_{p}-\left(\sin \Lambda_{x} \sin \Lambda_{y} R_{*} \cos \phi_{*} \sin \vartheta_{*}\right. \\
& \left.+\cos \Lambda_{x} R_{*} \sin \phi_{*} \sin \vartheta_{*}+\sin \Lambda_{x} \cos \Lambda_{y} R_{*} \cos \vartheta_{*}+y_{*}\right),
\end{aligned}
$$

$$
\begin{aligned}
\sigma_{z}= & R_{p} \cos \vartheta_{p}-\left(\cos \Lambda_{x} \sin \Lambda_{y} R_{*} \cos \phi_{*} \sin \vartheta_{*}\right. \\
& \left.-\sin \Lambda_{x} R_{*} \sin \phi_{*} \sin \vartheta_{*}+\cos \Lambda_{x} \cos \Lambda_{y} R_{*} \cos \vartheta_{*}+z_{*}\right)
\end{aligned}
$$

The unit vector in the direction of $\sigma$ is defined as

$\hat{\sigma}=\frac{1}{s} \sigma$,

where s is the distance between the cells $C_{\mathrm{p}}$ and $C_{*}$ (the square root of the sum of squares of the components of $\sigma$ given above).

The unit vector normal to the planet cell $\hat{n}_{\mathrm{p}}$ is

$\hat{\boldsymbol{n}}_{\mathrm{p}}=\left[\begin{array}{c}\cos \phi_{p} \sin \vartheta_{p} \\ \sin \phi_{p} \sin \vartheta_{p} \\ \cos \vartheta_{p}\end{array}\right]_{p}$

The dot product of the two unit vectors is

$$
\begin{aligned}
\hat{\sigma} . \hat{n}_{\mathrm{p}}= & \frac{1}{s}\left\{\operatorname { c o s } \phi _ { p } \operatorname { s i n } \vartheta _ { p } \left[R_{\mathrm{p}} \cos \phi_{p} \sin \vartheta_{p}\right.\right. \\
& \left.-\left(\cos \Lambda_{y} R_{*} \cos \phi_{*} \sin \vartheta_{*}-\sin \Lambda_{y} R_{*} \cos \vartheta_{*}+x_{*}\right)\right] \\
& +\sin \phi_{p} \sin \vartheta_{p}\left[R_{\mathrm{p}} \sin \phi_{*} \sin \vartheta_{p}\right. \\
& -\left(\sin \Lambda_{x} \sin \Lambda_{y} R_{*} \cos \phi_{*} \sin \vartheta_{*}\right. \\
& \left.\left.+\cos \Lambda_{x} R_{*} \sin \phi_{*} \sin \vartheta_{*}+\sin \Lambda_{x} \cos \Lambda_{y} R_{*} \cos \vartheta_{*}+y_{*}\right)\right] \\
& +\cos \vartheta_{p}\left[R_{\mathrm{p}} \cos \vartheta_{p}-\left(\cos \Lambda_{x} \sin \Lambda_{y} R_{*} \cos \phi_{*} \sin \vartheta_{*}\right.\right. \\
& \left.\left.-\sin \Lambda_{x} R_{*} \sin \phi_{*} \sin \vartheta_{*}+\cos \Lambda_{x} \cos \Lambda_{y} R_{*} \cos \vartheta_{*}+z_{*}\right]\right\} .
\end{aligned}
$$

Now, we determine the angles $\Lambda_{x}$ and $\Lambda_{y}$. We use equation (A14) and set the position of the star cell to be in the direction of the $Z_{*}^{\prime}$ axis and we set it to unit length. When this axis is rotated, it must 


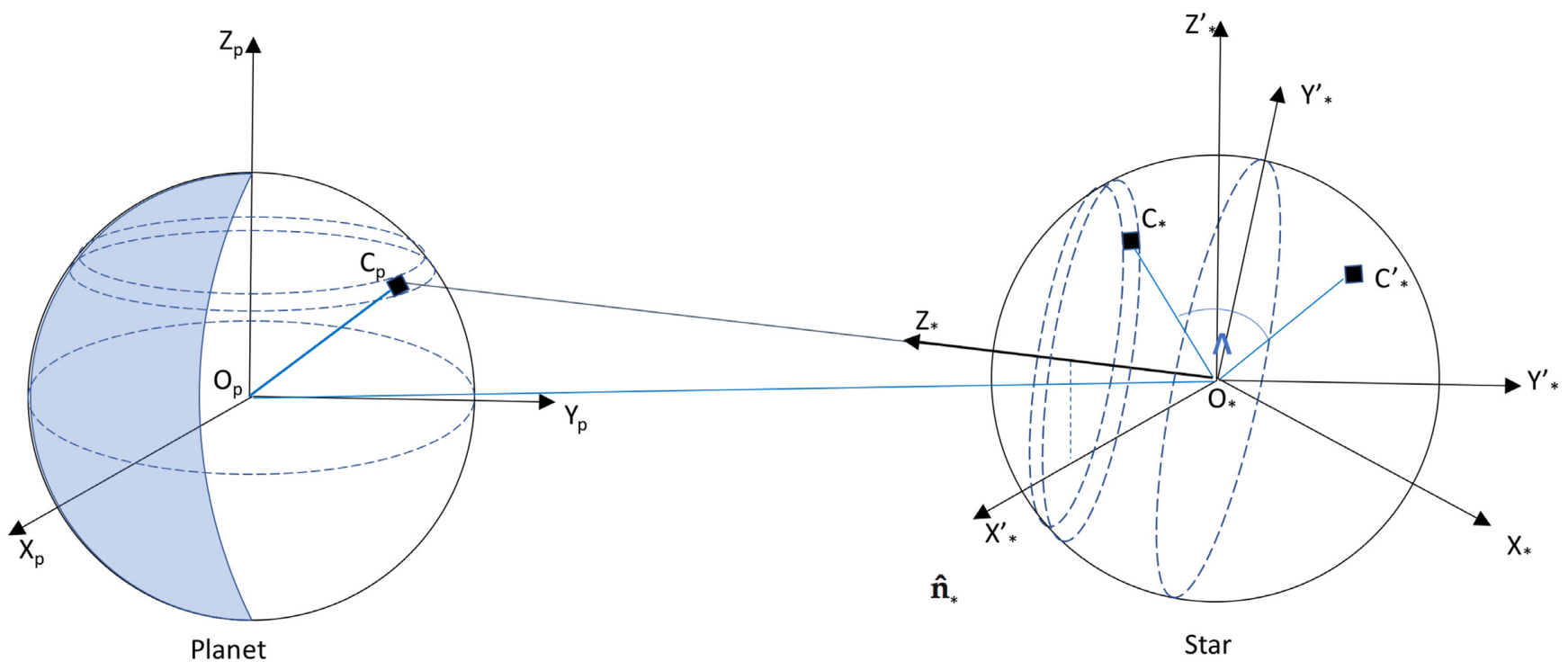

Figure A1. Geometry showing the rotation from the original star frame $X_{*}^{\prime} Y_{*}^{\prime} Z_{*}^{\prime}$ to the frame $X_{*} Y_{*} Z_{*}$ where the $Z_{*}$ axis points towards the planet cell $C_{\mathrm{p}}$.

be pointing towards the planet cell so we have

$$
\frac{1}{q}\left[\begin{array}{l}
C_{p x}-x_{*} \\
C_{p y}-y_{*} \\
C_{p z}-z_{*}
\end{array}\right]_{p}=\left[\begin{array}{ccc}
\cos \Lambda_{y} & 0 & -\sin \Lambda_{y} \\
\sin \Lambda_{x} \sin \Lambda_{y} & \cos \Lambda_{x} & \sin \Lambda_{x} \cos \Lambda_{y} \\
\cos \Lambda_{x} \sin \Lambda_{y} & -\sin \Lambda_{x} & \cos \Lambda_{x} \cos \Lambda_{y}
\end{array}\right]
$$$$
\times\left[\begin{array}{l}
0 \\
0 \\
1
\end{array}\right]_{*}
$$

where $\mathrm{q}$ is the normalizing factor

$$
\begin{aligned}
q^{2}= & \left(R_{\mathrm{p}} \cos \phi_{p} \sin \vartheta_{p}-x_{*}\right)^{2} \\
& +\left(R_{\mathrm{p}} \sin \phi_{p} \sin \vartheta_{p}-y_{*}\right)^{2}+\left(R_{\mathrm{p}} \cos \vartheta_{p}-z_{*}\right)^{2} .
\end{aligned}
$$

Simplifying the equation, we get

$\frac{1}{q}\left[\begin{array}{c}R_{\mathrm{p}} \cos \phi_{p} \sin \vartheta_{p}-x_{*} \\ R_{\mathrm{p}} \sin \phi_{p} \sin \vartheta_{p}-y_{*} \\ R_{\mathrm{p}} \cos \vartheta_{p}-z_{*}\end{array}\right]=\left[\begin{array}{c}-\sin \Lambda_{y} \\ \sin \Lambda_{x} \cos \Lambda_{y} \\ \cos \Lambda_{x} \cos \Lambda_{y}\end{array}\right]$,

and from this we have

$$
\begin{aligned}
& \Lambda_{y}=\sin ^{-1}\left(\frac{x_{*}-R_{\mathrm{p}} \cos \phi_{p} \sin \vartheta_{p}}{q}\right), \\
& \Lambda_{x}=\sin ^{-1}\left(\frac{R_{\mathrm{p}} \sin \phi_{p} \sin \vartheta_{p}-y_{*}}{q \cos \Lambda_{y}}\right) .
\end{aligned}
$$

The flux arriving at the surface of the planet at the point $\left(\vartheta_{\mathrm{p}}, \phi_{\mathrm{p}}, R_{\mathrm{p}}\right)$ is

$$
F_{\vartheta_{p} \phi_{p}}(v)=\int_{0}^{2 \pi} \int_{0}^{\pi / 2} H\left(\vartheta_{\mathrm{p}}, \phi_{\mathrm{p}}, \vartheta_{*}, \phi_{*}, v-\Delta v_{*}, t\right) \mathrm{d} \vartheta_{*} \mathrm{~d} \phi_{*},
$$

where $H\left(\vartheta_{\mathrm{p}}, \phi_{\mathrm{p}}, \vartheta_{*}, \phi_{*}, v, t\right)$ is zero when starlight is blocked from reaching the planet cell

$$
H\left(\vartheta_{\mathrm{p}}, \phi_{\mathrm{p}}, \vartheta_{*}, \phi_{*}, v, t\right)= \begin{cases}0, & \text { if } \hat{\sigma} \cdot \hat{n}_{*}<0 \\ 0, & \text { if }-\hat{\sigma} \cdot \hat{n}_{\mathrm{p}}<0,\end{cases}
$$

otherwise non-zero when

$$
\begin{aligned}
& H\left(\vartheta_{\mathrm{p}}, \phi_{\mathrm{p}}, \vartheta_{*}, \phi_{*}, v, t\right)=I_{*}\left(\vartheta_{*}, \phi_{*}, \nu, t\right)\left(-\hat{n}_{p} \cdot \hat{\sigma}\right) \\
& \quad \times\left(\frac{-R_{*} \sin \vartheta_{*}}{\sqrt{R_{*}^{2}+d^{2}-2 R_{*} d \cos \vartheta_{*}}}+\frac{R_{*} d \sin \vartheta_{*}\left(d-R_{*} \cos \vartheta_{*}\right)}{\left(R_{*}^{2}+d^{2}-2 d R_{*} \cos \vartheta_{*}\right)^{3 / 2}}\right) .
\end{aligned}
$$

The intensity of the reflected light leaving the surface from the planet cell $\left(\theta_{\mathrm{p}}, \phi_{\mathrm{p}}\right)$ in the direction of the Earth is

$$
\begin{aligned}
I_{s \vartheta_{\mathrm{p}} \phi_{\mathrm{p}}}(\nu)= & \int_{0}^{2 \pi} \int_{0}^{\pi / 2} \rho\left(\vartheta_{\mathrm{p}}, \phi_{\mathrm{p}}, \vartheta_{*}, \vartheta_{*}\right) \\
& \times H\left(\vartheta_{\mathrm{p}}, \phi_{\mathrm{p}}, \vartheta_{*}, \phi_{*}, v-\Delta v, t\right)\left(\hat{n}_{\mathrm{p}} \cdot \hat{k}\right) \mathrm{d} \vartheta_{*} \mathrm{~d} \phi_{*},
\end{aligned}
$$

where $\rho\left(\vartheta_{\mathrm{p}}, \phi_{\mathrm{p}}, \vartheta_{*}, \vartheta_{*}\right)$ is the bidirectional reflectance distribution function, $\hat{k}$ is the unit vector in the direction of the Earth, and the frequency Doppler shift $\Delta v$ is

$\frac{\Delta v}{v}=\frac{\Delta v}{c}$,

where $\Delta v$ is the velocity shift due to the reflection of the light on the cell $C_{\mathrm{p}}$ and the shift due to the radial motion of the star cell with respect to the planet cell $\Delta v_{r, c_{*} c_{\mathrm{p}}}$. This velocity shift is

$\Delta v=v_{\mathrm{p}} \cdot(\hat{k}-\hat{\sigma})+\Delta v_{r, c_{*} c_{\mathrm{p}}}$.

The surface velocity of the planet cell $v_{\mathrm{p}}$ is determined in Appendix $\mathrm{B}$ and $\Delta v_{r, c_{*} c_{\mathrm{p}}}$ is determined in Appendix $\mathrm{C}$.

We now work out the reflected starlight reaching the observer at the Earth. The viewpoint of the observer is given in Fig. A2 and from this we can see that $\mathrm{d} \Omega_{\mathrm{p}}$ and $\tan \omega$ is

$$
\begin{aligned}
& \mathrm{d} \Omega_{\mathrm{p}}=\frac{\mathrm{d} A}{r^{2}}=\sin \omega \mathrm{d} \omega \mathrm{d} \phi_{\mathrm{p}}, \\
& \tan \omega=\frac{\rho}{d_{\mathrm{p} \oplus}} .
\end{aligned}
$$

Assuming the Earth is very far from the planet we can treat $\omega$ as a small angle and Taylor expanding only to first order we have 


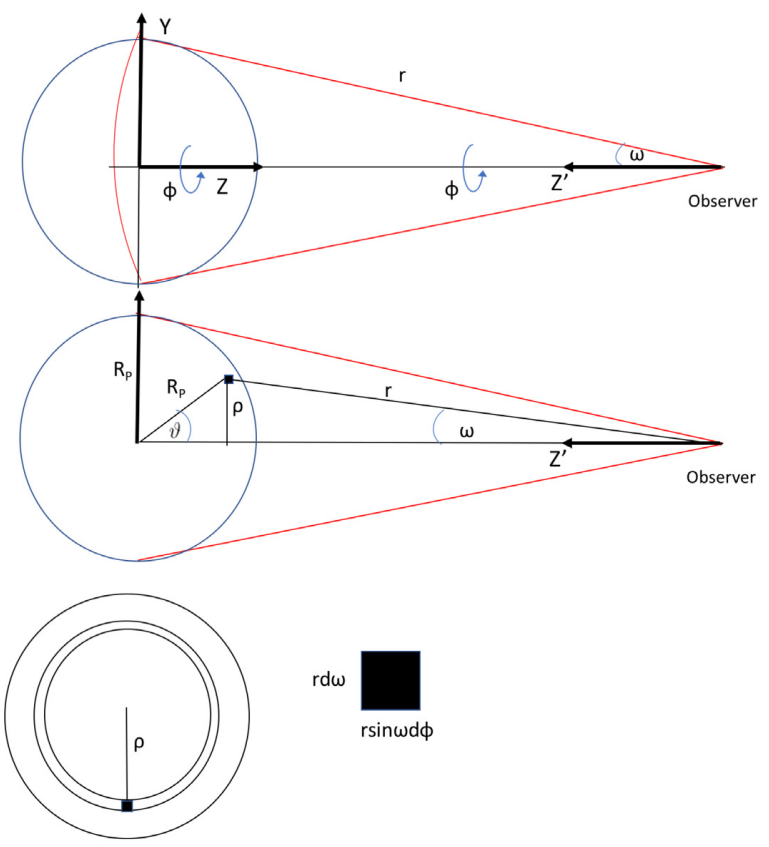

Figure A2. Geometry showing the view of the planet with respect to the observer.

$\sin \omega=\omega$ and $\cos \omega=1$ and thus

$\omega=\frac{R_{\mathrm{P}} \sin \vartheta_{\mathrm{p}}}{d_{\mathrm{p} \oplus}}$,

where $d_{\mathrm{p} \oplus}$ is distance from the Earth to the planet. Differentiating, we have

$\mathrm{d} \omega=\left(R_{\mathrm{P}} \cos \vartheta_{\mathrm{p}} / d_{\mathrm{p} \oplus}\right) \mathrm{d} \vartheta_{\mathrm{p}}$.

The flux arriving at the distant Earth is

$$
\begin{aligned}
F_{\oplus}(v) & =\int_{0}^{2 \pi} \int_{0}^{\frac{R_{\mathrm{p}}}{d_{p \oplus}}} I_{s \vartheta_{p} \phi_{p}}\left(\vartheta_{p}, \phi_{p}, v, t\right) \cos \omega \sin \omega \mathrm{d} \omega \mathrm{d} \phi_{p} \\
& =\int_{0}^{2 \pi} \int_{0}^{\frac{R_{\mathrm{p}}}{d_{\mathrm{p} \oplus}}} I_{s \vartheta_{p} \phi_{p}}\left(\vartheta_{p}, \phi_{p}, v, t\right) \omega \mathrm{d} \omega \mathrm{d} \phi_{p},
\end{aligned}
$$

and thus

$$
\begin{aligned}
F_{\oplus}(\nu, t)= & \left(\frac{R_{\mathrm{p}}}{d_{\mathrm{p} \oplus}}\right)^{2} \int_{0}^{2 \pi} \int_{0}^{\pi / 2} I_{s \vartheta_{p} \phi_{p}}\left(\vartheta_{p}, \phi_{p}, v, t\right) \\
& \times \sin \vartheta_{p} \cos \vartheta_{p} \mathrm{~d} \vartheta_{p} \mathrm{~d} \phi_{p} .
\end{aligned}
$$

\section{APPENDIX B: POSITION AND VELOCITY OF POINTS ON SURFACE OF THE PLANET}

\section{B1 Introduction}

Initially, we work in the frame centred on the barycentre and with $x y$ plane coincident with the plane of the orbit and measure position and velocity of points on the surface of the planet relative to the barycentre. We then move on to determining these velocities in the reference frame as is typically viewed from the Earth using circularized forms of the elliptical two-body equations in order to transform from the barycentric frame to the Earth frame.

The planet moves round the barycentre in a circle or radius a in $x y$ plane with coordinates

$$
\begin{aligned}
& x=a \frac{m_{*}}{m_{*}+m_{\mathrm{p}}} \cos \theta, \\
& y=a \frac{m_{*}}{m_{*}+m_{\mathrm{p}}} \sin \theta .
\end{aligned}
$$

Fig. B1 shows the path of the orbit of the planet and star and that of the surface cells around the barycentre in the $x y$ plane.

The coordinates of the planet are $P=\left(x_{\mathrm{p}}, y_{\mathrm{p}}, 0\right)$ and surface cell coordinates are $C_{\mathrm{p}}=\left(x_{\mathrm{pc}}, y_{\mathrm{pc}}, z_{\mathrm{pc}}\right)$.

From Fig. 14, it is clear that the cell $C_{\mathrm{p}}$ moves in a circular orbit with radius

$d_{\mathrm{pc}}=\sqrt{x_{\mathrm{pc}}^{2}+y_{\mathrm{pc}}^{2}}$,

at a height $z_{\mathrm{pc}}$ above the $x y$ plane. We can write the $x$ and $y$ coordinates of the planet in terms of $\theta$ and $d_{\mathrm{pc}}$ as

$x_{\mathrm{pc}}=d_{\mathrm{pc}} \cos \left(\theta+\theta_{\mathrm{pc}}^{\prime}\right)$,
$y_{\mathrm{pc}}=d_{\mathrm{pc}} \sin \left(\theta+\theta_{\mathrm{pc}}^{\prime}\right)$,
where

$\sin \theta_{\mathrm{pc}}^{\prime}=\frac{\mathrm{BP} \times\left(x_{\mathrm{pc}}, y_{\mathrm{pc}}, 0\right)^{T}}{|\mathrm{BP}|\left|\left(x_{\mathrm{pc}}, y_{\mathrm{pc}}, 0\right)\right|}$,

and $\theta_{\mathrm{pc}}^{\prime}$ is in range $\{-\pi / 2, \pi / 2\}$.

Differentiating, we get the velocity of the cell in the frame to be

$$
\begin{aligned}
& \dot{x}_{\mathrm{pc}}=-d_{\mathrm{pc}} \dot{\theta} \sin \left(\theta+\theta_{\mathrm{pc}}^{\prime}\right), \\
& \dot{y}_{\mathrm{pc}}=d_{\mathrm{pc}} \dot{\theta} \cos \left(\theta+\theta_{\mathrm{pc}}^{\prime}\right), \\
& \dot{z}_{\mathrm{pc}}=0 .
\end{aligned}
$$

We can now move to the barycentric frame with $Z$-axis pointing to the Earth and the $X$ - and $Y$ - axes in the plane of the sky

$$
\begin{aligned}
& {\left[\begin{array}{l}
X_{\mathrm{pc}} \\
Y_{\mathrm{pc}} \\
Z_{\mathrm{pc}}
\end{array}\right]=\left[\begin{array}{ccc}
\cos \Omega & -\sin \Omega & 0 \\
\sin \Omega & \cos \Omega & 0 \\
0 & 0 & 1
\end{array}\right]\left[\begin{array}{ccc}
1 & 0 & 0 \\
0 & \cos I & -\sin I \\
0 & \sin I & \cos I
\end{array}\right]} \\
& \times\left[\begin{array}{c}
d_{\mathrm{pc}} \cos \left(\theta+\theta_{\mathrm{pc}}^{\prime}\right) \\
d_{\mathrm{pc}} \sin \left(\theta+\theta_{\mathrm{pc}}^{\prime}\right) \\
z_{\mathrm{pc}}
\end{array}\right], \\
& {\left[\begin{array}{c}
X_{\mathrm{pc}} \\
Y_{\mathrm{pc}} \\
Z_{\mathrm{pc}}
\end{array}\right]=\left[\begin{array}{ccc}
\cos \Omega & -\sin \Omega & 0 \\
\sin \Omega & \cos \Omega & 0 \\
0 & 0 & 1
\end{array}\right]} \\
& \times\left[\begin{array}{c}
d_{\mathrm{pc}} \cos \left(\theta+\theta_{\mathrm{pc}}^{\prime}\right) \\
d_{\mathrm{pc}} \sin \left(\theta+\theta_{\mathrm{pc}}^{\prime}\right) \cos I-z_{\mathrm{pc}} \sin I \\
d_{\mathrm{pc}} \sin \left(\theta+\theta_{\mathrm{pc}}^{\prime}\right) \sin I+z_{\mathrm{pc}} \cos I
\end{array}\right],
\end{aligned}
$$

$\left[\begin{array}{c}X_{\mathrm{pc}} \\ Y_{\mathrm{pc}} \\ Z_{\mathrm{pc}}\end{array}\right]$

$$
=\left[\begin{array}{c}
d_{\mathrm{pc}}\left(\cos \Omega \cos \left(\theta+\theta_{\mathrm{pc}}^{\prime}\right)-\sin \Omega \sin \left(\theta+\theta_{\mathrm{pc}}^{\prime}\right) \cos I\right)+z_{\mathrm{pc}} \sin \Omega \sin I \\
d_{\mathrm{pc}}\left(\sin \Omega \cos \left(\theta+\theta_{\mathrm{pc}}^{\prime}\right)+\cos \Omega \sin \left(\theta+\theta_{\mathrm{pc}}^{\prime}\right) \cos I\right)-z_{\mathrm{pc}} \cos \Omega \sin I \\
d_{\mathrm{pc}} \sin \left(\theta+\theta_{\mathrm{pc}}^{\prime}\right) \sin I+z_{\mathrm{pc}} \cos I
\end{array}\right] .
$$




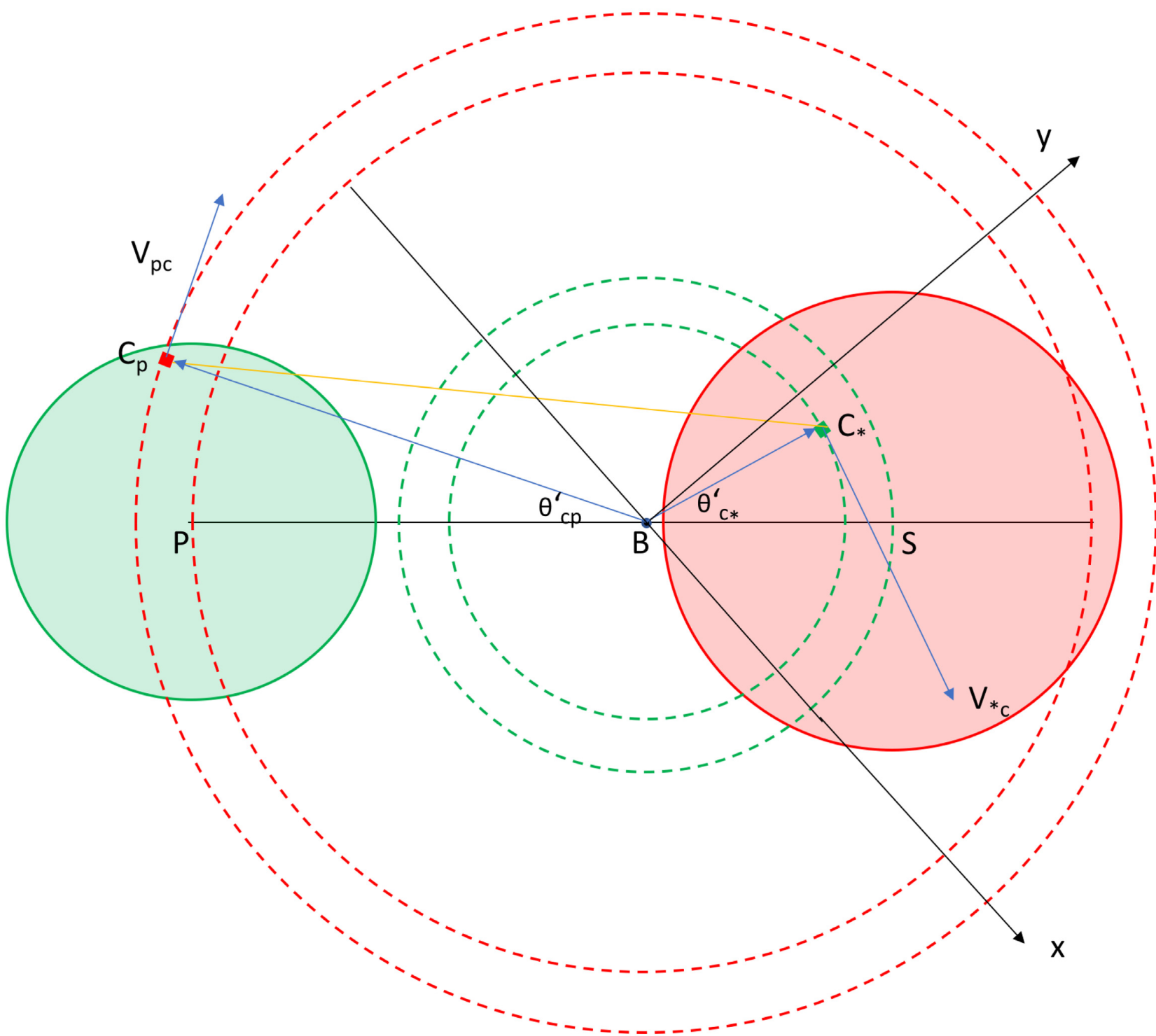

Figure B1. Geometry looking down on $x y$ plane of planet and star orbiting the barycentre B along with the paths and motion of cells on the surface of the planet and the star.

Differentiating, we get the velocity

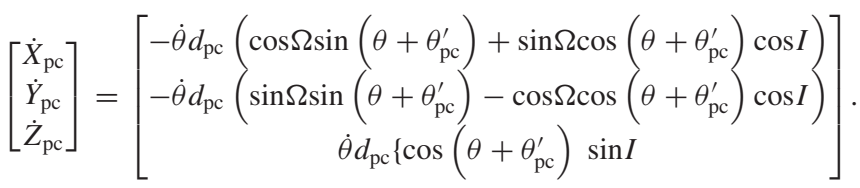

We typically know $\Omega, I$ and $\left\{X_{\mathrm{pc}}, Y_{\mathrm{pc}}, Z_{\mathrm{pc}}\right\}^{T}$ and $\theta$ and need to determine $d_{\mathrm{pc}}, z_{\mathrm{pc}}$, and $\theta_{\mathrm{pc}}^{\prime}$ from the above equations. Noting that the above equations are linear in $d_{\mathrm{pc}} \cos (\theta+$ $\left.\theta_{\mathrm{pc}}^{\prime}\right), d_{\mathrm{pc}} \sin \left(\theta+\theta_{\mathrm{pc}}^{\prime}\right)$ and $z_{\mathrm{pc}}$, we can arrange the matrices as follows:

$$
\begin{aligned}
{\left[\begin{array}{c}
X_{\mathrm{pc}} \\
Y_{\mathrm{pc}} \\
Z_{\mathrm{pc}}
\end{array}\right]=} & {\left[\begin{array}{ccc}
\cos \Omega & -\sin \Omega \cos I & \sin \Omega \sin I \\
\sin \Omega & \cos \Omega \cos I & -\cos \Omega \sin I \\
0 & \sin I & \cos I
\end{array}\right] } \\
& \times\left[\begin{array}{c}
d_{\mathrm{pc}} \cos \left(\theta+\theta_{\mathrm{pc}}^{\prime}\right) \\
d_{\mathrm{pc}} \sin \left(\theta+\theta_{\mathrm{pc}}^{\prime}\right) \\
z_{\mathrm{pc}}
\end{array}\right] .
\end{aligned}
$$


We can invert the above matrix (determinant is 1 so it is nonsingular) and solve for our three parameters:

$$
\begin{aligned}
{\left[\begin{array}{c}
d_{\mathrm{pc}} \cos \left(\theta+\theta_{\mathrm{pc}}^{\prime}\right) \\
d_{\mathrm{pc}} \sin \left(\theta+\theta_{\mathrm{pc}}^{\prime}\right) \\
z_{\mathrm{pc}}
\end{array}\right]=} & {\left[\begin{array}{c}
\cos \Omega \\
-\sin \Omega \cos I \\
\sin \Omega \sin I
\end{array}\right.} \\
& \times\left[\begin{array}{c}
X_{\mathrm{pc}} \\
Y_{\mathrm{pc}} \\
Z_{\mathrm{pc}}
\end{array}\right]=\left[\begin{array}{c}
l \\
m \\
n
\end{array}\right] .
\end{aligned}
$$

$\sin \Omega \quad 0$ $\cos \Omega \cos I \quad \sin I$ $-\cos \Omega \sin I \quad \cos I]$

From this, we can see that

$d_{\mathrm{pc}}=\sqrt{l^{2}+m^{2}}$,

and

$\sin \theta^{\prime}=\frac{m \cos \theta-l \sin \theta}{d_{\mathrm{pc}}}$.

\section{APPENDIX C: POSITION AND VELOCITY OF POINT ON SURFACE OF THE STAR}

We determine the velocity and position of a point on the surface of the star - a star cell - with respect to the barycentre of the system.

The velocity of a star cell $v_{* c}$ is formed of two components due to the motion of the orbit of the star around the centre of mass of the system $v_{* c, o}$ and the spin of the star $v_{* c \text {, sp }}$

$v_{* c}=v_{* c, o}+v_{* c, \mathrm{sp}}$.

In our determination of the Doppler shift of reflected light, we need to know the component of the surface velocity in the direction of a given planet cell. If we denote $(\hat{\sigma})$ as the unit vector in that direction then the component velocity is given by

$\Delta v_{r, c_{*} c_{p}}=v_{* c} \cdot \hat{\sigma}$.

The next two subsections determine each of the terms on the right-hand side of equation $(\mathrm{C} 1)$ in turn.

\section{C1 Orbital component of velocity of star cell}

The star moves round the barycentre in a circle of radius a in the $x y$ plane with coordinates

$x=a \frac{m_{\mathrm{p}}}{m_{*}+m_{\mathrm{p}}} \cos \theta$,

$y=a \frac{m_{p}}{m_{*}+m_{p}} \sin \theta$.

Fig. B1 shows the path of the orbit of the planet and star and that of the surface cells around the barycentre in the $x y$ plane. The coordinates of the star are $P=\left(x_{*}, y_{*}, 0\right)$ and surface cell coords is $C_{\mathrm{p}}=\left(x_{* c}, y_{* c}, z_{* c}\right)$. From B1, it is clear that the cell $C_{*}$ moves in a circular orbit with radius

$d_{* c}=\sqrt{x_{* c}^{2}+y_{* c}^{2}}$,

at a height $z_{* c}$ above the $x y$ plane. We can write the $x$ and $y$ coordinates of the planet in terms of $\theta$ and $d_{* c}$ as

$x_{* c}=d_{* c} \cos \left(\theta+\theta_{* c}^{\prime}+\pi\right)=-d_{* c} \cos \left(\theta+\theta_{* c}^{\prime}\right)$,

$y_{* c}=d_{* c} \sin \left(\theta+\theta_{* c}^{\prime}+\pi\right)=-d_{* c} \sin \left(\theta+\theta_{* c}^{\prime}\right)$,

$z_{* c}=z_{* c}$, where

$\sin \theta_{* c}^{\prime}=\frac{B S \times\left(x_{* c}, y_{* c}, 0\right)^{T}}{|B S|\left|\left(x_{* c}, y_{* c}, 0\right)\right|}$

and $\theta_{* c}^{\prime}$ is in range $\{-\pi / 2, \pi / 2\}$.

Differentiating, we get the velocity of the cell in the frame to be

$\dot{x}_{* c}=d_{* c} \dot{\theta} \sin \left(\theta+\theta_{* c}^{\prime}\right)$,

$\dot{y}_{* c}=-d_{* c} \dot{\theta} \cos \left(\theta+\theta_{* c}^{\prime}\right)$,

$\dot{z}_{* c}=0$.

We can now move to the barycentric frame with $Z$-axis pointing to the Earth and the $X$ and $Y$ axis in the plane of the sky:

$$
\begin{aligned}
{\left[\begin{array}{l}
X_{* c} \\
Y_{* c} \\
Z_{* c}
\end{array}\right]=} & {\left[\begin{array}{ccr}
\cos \Omega & -\sin \Omega & 0 \\
\sin \Omega & \cos \Omega & 0 \\
0 & 0 & 1
\end{array}\right] } \\
& \times\left[\begin{array}{ccc}
1 & 0 & 0 \\
0 & \cos I & -\sin I \\
0 & \sin I & \cos I
\end{array}\right]\left[\begin{array}{c}
-d_{* c} \cos \left(\theta+\theta_{\mathrm{pc}}^{\prime}\right) \\
-d_{* c} \sin \left(\theta+\theta_{\mathrm{pc}}^{\prime}\right) \\
z_{* c}
\end{array}\right],
\end{aligned}
$$

$$
\begin{aligned}
{\left[\begin{array}{l}
X_{* c} \\
Y_{* c} \\
Z_{* c}
\end{array}\right]=} & {\left[\begin{array}{ccr}
\cos \Omega & -\sin \Omega & 0 \\
\sin \Omega & \cos \Omega & 0 \\
0 & 0 & 1
\end{array}\right] } \\
\times & {\left[\begin{array}{c}
-d_{* c} \cos \left(\theta+\theta_{* c}^{\prime}\right) \\
-d_{* c} \sin \left(\theta+\theta_{* c}^{\prime}\right) \cos I-z_{* c} \sin I \\
-d_{* c} \sin \left(\theta+\theta_{* c}^{\prime}\right) \sin I+z_{* c} \cos I
\end{array}\right], }
\end{aligned}
$$

$$
\begin{gathered}
{\left[\begin{array}{c}
X_{* c} \\
Y_{* c} \\
Z_{* c}
\end{array}\right]} \\
=\left[\begin{array}{c}
-d_{* c}\left(\cos \Omega \cos \left(\theta+\theta_{* c}^{\prime}\right)-\sin \Omega \sin \left(\theta+\theta_{* c}^{\prime}\right) \cos I\right)+z_{* c} \sin \Omega \sin I \\
-d_{* c}\left(\sin \Omega \cos \left(\theta+\theta_{* c}^{\prime}\right)+\cos \Omega \sin \left(\theta+\theta_{* c}^{\prime}\right) \cos I\right)-z_{* c} \cos \Omega \sin I \\
-d_{* c} \sin \left(\theta+\theta_{* c}^{\prime}\right) \sin I+z_{* c} \cos I
\end{array}\right] .
\end{gathered}
$$

Differentiating, we get the velocity

$v_{* c, o}=\left[\begin{array}{c}\dot{X}_{* c} \\ \dot{Y}_{* c} \\ \dot{Z}_{* c}\end{array}\right]$

$$
v_{* c, o}=\left[\begin{array}{c}
\dot{\theta} d_{* c}\left(\cos \Omega \sin \left(\theta+\theta_{* c}^{\prime}\right)+\sin \Omega \cos \left(\theta+\theta_{* c}^{\prime}\right) \cos I\right) \\
\dot{\theta} d_{* c}\left(\sin \Omega \sin \left(\theta+\theta_{* c}^{\prime}\right)-\cos \Omega \cos \left(\theta+\theta_{* c}^{\prime}\right) \cos I\right) \\
-\dot{\theta} d_{* c} \cos \left(\theta+\theta_{* c}^{\prime}\right) \sin I
\end{array}\right] .
$$

We typically know $\Omega, I$ and $\left\{X_{* c}, Y_{* c}, Z_{* c}\right\}^{T}$ and $\theta$ and need to determine $d_{* c}, z_{* c}$ and $\theta_{* c}^{\prime}$ from the above equations. Noting that the 
above equations are linear in $d_{* c} \cos \left(\theta+\theta_{* c}^{\prime}\right), d_{* c} \sin \left(\theta+\theta_{* c}^{\prime}\right)$ and $z_{* c}$, we can arrange the matrices as follows:

$$
\begin{aligned}
{\left[\begin{array}{c}
X_{* c} \\
Y_{* c} \\
Z_{* c}
\end{array}\right]=} & {\left[\begin{array}{ccc}
\cos \Omega & -\sin \Omega \cos I & \sin \Omega \sin I \\
\sin \Omega & \cos \Omega \cos I & -\cos \Omega \sin I \\
0 & \sin I & \cos I
\end{array}\right] } \\
& \times\left[\begin{array}{c}
-d_{* c} \cos \left(\theta+\theta_{* c}^{\prime}\right) \\
-d_{* c} \sin \left(\theta+\theta_{* c}^{\prime}\right) \\
z_{* c}
\end{array}\right] .
\end{aligned}
$$

We can invert the above matrix (determinant is 1 so it is nonsingular) and solve for our three parameters:

$$
\begin{aligned}
{\left[\begin{array}{c}
-d_{* c} \cos \left(\theta+\theta_{* c}^{\prime}\right) \\
-d_{* c} \sin \left(\theta+\theta_{* c}^{\prime}\right) \\
z_{* c}
\end{array}\right]=} & {\left[\begin{array}{ccc}
\cos \Omega & \sin \Omega & 0 \\
-\sin \Omega \cos I & \cos \Omega \cos I & \sin I \\
\sin \Omega \sin I & -\cos \Omega \sin I & \cos I
\end{array}\right] } \\
& \times\left[\begin{array}{l}
X_{* c} \\
Y_{* c} \\
Z_{* c}
\end{array}\right]=\left[\begin{array}{l}
l \\
m \\
n
\end{array}\right] .
\end{aligned}
$$

From this, we can see that

$d_{* c}=\sqrt{l^{2}+m^{2}}$,

and

$$
\sin \theta^{\prime}=\frac{l \sin \theta-m \cos \theta}{d_{* c}}
$$

\section{C2 Star cell velocity component due to spin of star}

Now, we determine the velocity of the star cell due to the spin of

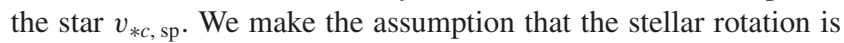
aligned to its orbit and in particular that there is no obliquity. We also assume that the observed rotation period of the star has been determined from photometry $P_{* \text {, obs }}$ and is related to the observed angular velocity $\Omega_{* \text {, obs }}$ as

$\Omega_{*, \mathrm{obs}}=\frac{2 \pi}{P_{*, \mathrm{obs}}}$.

The observed angular velocity is made up of two components due to the spin of the star and the orbit of the system:

$\Omega_{*, \mathrm{obs}}=\Omega_{*}+\Omega_{*, \mathrm{orb}}$

The magnitude of $\Omega_{* \text {, orb }}$ is known from the period of the exoplanet and star from the RV measurements, so we can determine the spin from the star $\Omega_{*}$. We determine the spin axis unit vector by noting that it is in the same direction out of the plane of orbit of the two-body model centred on the planet. Thus, we have

$\hat{\Omega}_{*}=\frac{r \times \dot{r}}{|r \times \dot{r}|}$.

Components or $\mathrm{r}$ and $\dot{r}$ with respect to the planet are $x=a \cos (\theta+\pi)=-a \cos \theta$,

$y=a \sin (\theta+\pi)=-a \sin \theta$,

$z=0$.

Transforming to viewpoint of the Earth in $X Y Z$ frame we have

$r=\left[\begin{array}{l}X_{* \mathrm{p}} \\ Y_{* \mathrm{p}} \\ Z_{* \mathrm{p}}\end{array}\right]=\left[\begin{array}{ccc}\cos \Omega & -\sin \Omega & 0 \\ \sin \Omega & \cos \Omega & 0 \\ 0 & 0 & 1\end{array}\right]\left[\begin{array}{ccc}1 & 0 & 0 \\ 0 & \cos I & -\sin I \\ 0 & \sin I & \cos I\end{array}\right]$

$$
\times\left[\begin{array}{c}
-a \cos \theta \\
-a \sin \theta \\
0
\end{array}\right]
$$

$r=\left[\begin{array}{l}X_{* c} \\ Y_{* c} \\ Z_{* c}\end{array}\right]=\left[\begin{array}{ccc}\cos \Omega & -\sin \Omega & 0 \\ \sin \Omega & \cos \Omega & 0 \\ 0 & 0 & 1\end{array}\right]\left[\begin{array}{c}-a \cos \theta \\ -a \sin \theta \cos I \\ -a \sin \theta \sin I\end{array}\right]$,

$r=\left[\begin{array}{c}X_{* p} \\ Y_{* p} \\ Z_{* p}\end{array}\right]=\left[\begin{array}{c}-a(\cos \Omega \cos \theta-\sin \Omega \sin \theta \cos I) \\ -a(\sin \Omega \cos \theta+\cos \Omega \sin \theta \cos I) \\ -a \sin \theta \sin I\end{array}\right]$.

Differentiating, we get the velocity

$\dot{r}=\left[\begin{array}{c}\dot{X}_{* p} \\ \dot{Y}_{* p} \\ \dot{Z}_{* p}\end{array}\right]=\left[\begin{array}{c}\dot{\theta} a(\cos \Omega \sin \theta+\sin \Omega \cos \theta \cos I) \\ \dot{\theta} a(\sin \Omega \sin \theta-\cos \Omega \cos \theta \cos I) \\ -\dot{\theta} a \cos \theta \sin I\end{array}\right]$.

Now, the cross product is

$r \times \dot{r}=a^{2} \dot{\theta}\left[\begin{array}{c}\sin I \sin \Omega \\ -\sin I \cos \Omega \\ \cos I\end{array}\right]$.

Dividing out the leading term $a^{2} \dot{\theta}$, we get the unit vector for $\hat{\Omega}_{*}$ to be

$\hat{\Omega}_{*}=\left[\begin{array}{c}\sin I \sin \Omega \\ -\sin I \cos \Omega \\ \cos I\end{array}\right]$.

If we let $r_{* c}$ denote the vector $\overrightarrow{O_{*} C_{*}}$ from the centre of the star to the star cell, then we have that the velocity of the star cell $v_{C_{*}}$ is just

$v_{* c, \mathrm{sp}}=\Omega_{*} \times r_{* c}$.

This paper has been typeset from a $\mathrm{T}_{\mathrm{E}} \mathrm{X} / \mathrm{L} \mathrm{T} \mathrm{E} \mathrm{X}$ file prepared by the author. 